\title{
The Ability to Acquire Iron Is Inversely Related to Virulence and the Protective Efficacy of Francisella tularensis Live Vaccine Strain
}

\section{OPEN ACCESS}

Edited by:

Leonard Peruski,

Centers for Disease Control and Prevention (CDC), United States

Reviewed by: Anders Sjöstedt, Umeå University, Sweden

Nagendran Tharmalingam, Alpert Medical School, Brown University, United States

${ }^{*}$ Correspondence: Bradley D. Jones bradley-jones@uiowa.edu

Specialty section: This article was submitted to Infectious Diseases,

a section of the journal

Frontiers in Microbiology

Received: 08 December 2017 Accepted: 15 March 2018 Published: 04 April 2018

Citation:

Fletcher JR, Crane DD, Wehrly TD, Martens $C A$, Bosio CM and Jones $B D$

(2018) The Ability to Acquire Iron Is Inversely Related to Virulence and the

Protective Efficacy of Francisella tularensis Live Vaccine Strain. Front. Microbiol. 9:607. doi: 10.3389/fmicb.2018.00607

\author{
Joshua R. Fletcher ${ }^{1}$, Deborah D. Crane ${ }^{2}$, Tara D. Wehrly ${ }^{2}$, Craig A. Martens ${ }^{3}$, \\ Catharine M. Bosio ${ }^{2}$ and Bradley D. Jones ${ }^{1,4 *}$
}

${ }^{1}$ Graduate Program in Genetics, University of lowa, lowa City, IA, United States, ${ }^{2}$ Immunity to Pulmonary Pathogens Section, Laboratory of Intracellular Parasites, Hamilton, MT, United States, ${ }^{3}$ Genomics Core, Research Technologies Branch, Rocky Mountain Laboratories, National Institute of Allergy and Infectious Diseases (NIAID), National Institutes of Health, Hamilton, MT, United States, ${ }^{4}$ Department of Microbiology, University of lowa, lowa City, IA, United States

Francisella tularensis is a highly infectious bacterial pathogen that causes the potentially fatal disease tularemia. The Live Vaccine Strain (LVS) of $F$. tularensis subsp. holarctica, while no longer licensed as a vaccine, is used as a model organism for identifying correlates of immunity and bacterial factors that mediate a productive immune response against F. tularensis. Recently, it was reported that two biovars of LVS differed in their virulence and vaccine efficacy. Genetic analysis showed that they differ in ferrous iron homeostasis; lower $\mathrm{Fe}^{2+}$ levels contributed to increased resistance to hydrogen peroxide in the vaccine efficacious LVS biovar. This also correlated with resistance to the bactericidal activity of interferon $\gamma$-stimulated murine bone marrow-derived macrophages. We have extended these findings further by showing that a mutant lacking bacterioferritin stimulates poor protection against Schu S4 challenge in a mouse model of tularemia. Together these results suggest that the efficacious biovar of LVS stimulates productive immunity by a mechanism that is dependent on its ability to limit the toxic effects of oxidative stress by maintaining optimally low levels of intracellular $\mathrm{Fe}^{2+}$.

Keywords: Francisella tularensis, iron acquisition mechanism, microbial pathogenesis, inflammatory mechanisms, virulence mechanisms, intracellular pathogen

\section{INTRODUCTION}

Francisella tularensis is a highly infectious bacterial pathogen that parasitizes the cytosol of host cells and causes the lethal disease tularemia in humans. The low infectious dose and high morbidity and mortality associated with tularemia have led the United States Centers for Disease Control and Prevention to designate F. tularensis a Tier 1 Select Agent. Fear of the intentional misuse of F. tularensis has spurred research into understanding the pathogenesis of the organism and the development of a vaccine. No licensed vaccine for $F$. tularensis infection is currently available for widespread use, although, the F. tularensis Live Vaccine Strain (LVS) has, in recent years, been used to vaccinate laboratory staff in the US and in other countries and large numbers of individuals have been vaccinated in the past (Eigelsbach and Downs, 1961). LVS is an attenuated derivative of F. tularensis subsp. holarctica that was generated in the Soviet Union in the mid- twentieth century but an incomplete understanding of the genetic changes, and therefore the underlying mechanisms, leading to the attenuation of the strain have led to its discontinuation as a licensed 
vaccine. Although not available as a human vaccine, LVS is routinely used as a model to understand the genetic requirements of a F. tularensis strain to stimulate host immunity and as a tool to discover and characterize correlates of immunity in the host. Each of these areas of understanding are critical in the rational design of a future vaccine against virulent $F$. tularensis species.

Despite significant differences in virulence, members of the Francisella genus are very similar at the genetic level; many of the observed differences are genomic rearrangements and single nucleotide polymorphisms (SNPs) (Rohmer et al., 2006, 2007). The genome similarities of the Francisella strains have made identification of specific factors that mediate the high level of virulence displayed by Type A and Type B strains difficult, although genome comparisons between F. tularensis subsp. tularensis Schu S4 and less virulent LVS have identified some of the attenuating mutations. These mutations include fusion of the $f u p A / B$ genes and deletion of pilA (Lindgren et al., 2009). Another noted difference between the highly virulent Type A strains and the intermediately virulent type B strains (including LVS) is the levels of intracellular iron, with virulence being inversely correlated with iron levels (lower intracellular iron correlates with higher virulence and higher intracellular iron correlates with lower virulence) (Lindgren et al., 2011). Iron is an essential micronutrient for bacteria, and numerous studies have shown that it is highly sought after by bacterial pathogens, as host sequestration of iron (a part of nutritional immunity) can restrict the growth of numerous pathogens (Cassat and Skaar, 2013; Parrow et al., 2013). Apparently, the virulence strategy of $F$. tularensis represents an exception to this paradigm as Lindgren et al., found that higher virulence subspecies had lower levels of intracellular iron (Lindgren et al., 2011).

Francisella species import iron via siderophore-bound ferric iron via the Fsl and Fup systems, and ferrous iron via FeoB and FupA/B; the latter system is unique to LVS (Ramakrishnan et al., 2008; Thomas-Charles et al., 2013; Perez and Ramakrishnan, 2014; Ramakrishnan and Sen, 2014). The feo operon typically consists of $f e o A B$, and less frequently feo $A B C$; the Francisella chromosome encodes $f e o A$ and $f e o B$ separately, and lacks $f e o C$ (Cartron et al., 2006; Perez and Ramakrishnan, 2014). FeoB is a large transmembrane protein that has an N-terminal Gprotein domain and a multi-pass transmembrane C-terminal domain (Marlovits et al., 2002; Andrews et al., 2003; Hantke, 2003). In other organisms, the $\mathrm{Fe}^{2+}$ import activity of FeoB requires interaction with the small protein FeoA, though the details of how FeoA stimulates FeoB activity are unknown (Su et al., 2010; Kim et al., 2012; Lau et al., 2013; Weaver et al., 2013). The general importance of iron in bacterial pathogenesis is reflected by the numerous ways that bacterial pathogens have evolved to obtain iron in a host, and several research groups have demonstrated that $f e o B$ contributes to or is required for full pathogenesis of Salmonella, Campylobacter, Helicobacter, Legionella, and others (Velayudhan et al., 2000; Robey and Cianciotto, 2002; Naikare et al., 2006; Aranda et al., 2009; Cassat and Skaar, 2013; Nagy et al., 2014). Multiple studies have characterized the $\mathrm{Fe}^{2+}$ uptake function of the F. tularensis $\mathrm{FeoB}$ and have linked this to its pathogenesis
(Thomas-Charles et al., 2013; Perez and Ramakrishnan, 2014; Lindgren et al., 2015). However, it is not clear from these studies if the modulation of growth among $f e o B$ mutants is a direct result of differential ability to use and take up iron or if there are indirect effects of excess iron that impact bacterial replication. For example, while iron is necessary, excess $\mathrm{Fe}^{2+}$ can contribute to toxicity for an organism as ferrous iron can participate in the Fenton reaction with $\mathrm{H}_{2} \mathrm{O}_{2}$, poisoning ironsulfur cluster enzymes in essential metabolic pathways (Imlay, 2013).

Francisella tularensis virulence is connected to the ability of the organism to avoid the detrimental effects of reactive oxygen species (ROS), such as $\mathrm{H}_{2} \mathrm{O}_{2}$ (Bou-Abdallah et al., 2002; Lindgren et al., 2007; McCaffrey et al., 2010; Lindemann et al., 2011; Crane et al., 2014; Ma et al., 2014). The bacterium couples glutamate metabolic pathways to $\mathrm{H}_{2} \mathrm{O}_{2}$ neutralization, and also maintains optimally low levels of intracellular $\mathrm{Fe}^{2+}$ such that Fenton reaction-mediated damage appears to be minimized (Lindgren et al., 2011; Ramond et al., 2014). When this optimal iron threshold is surpassed, numerous antioxidant enzymes are employed to protect the organism from toxic $\mathrm{H}_{2} \mathrm{O}_{2}$; mutants lacking enzymes like the Dyp peroxidase, superoxide dismutase and catalase are more sensitive to $\mathrm{H}_{2} \mathrm{O}_{2}$ damage, fail to inhibit host inflammatory signaling, and are often attenuated in murine infection models (Bakshi et al., 2006; Melillo et al., 2009, 2010; Llewellyn et al., 2011; Binesse et al., 2015; Rabadi et al., 2015; Shakerley et al., 2015). A growing body of literature has shown that F. tularensis utilizes numerous strategies to avoid activation of ROS-dependent host signaling pathways and killing by host-generated reactive oxygen and reactive nitrogen species (Buchan et al., 2009; Melillo et al., 2009, 2010; McKenna et al., 2010; Langmead and Salzberg, 2012; Binesse et al., 2015; Griffin et al., 2015; Rabadi et al., 2015; Shakerley et al., 2015). Furthermore, at least one phenotypic difference between the virulent $F$. tularensis Schu S4 strain and the non-pathogenic F. novicida strain is that the latter is considerably more sensitive to $\mathrm{H}_{2} \mathrm{O}_{2}$; this sensitivity is associated with activation of the AIM2 inflammasome (Zubay et al., 1972).

In this work we describe how regulation of iron uptake by F. tularensis LVS has a critical impact on the mouse virulence of the organism and on the resulting ability of the strain to induce effective protection against challenge with virulent F. tularensis. We first demonstrate how genetic variability in FeoB-mediated $\mathrm{Fe}^{2+}$ uptake observed in low vs. highly efficacious strains of LVS contributes to $\mathrm{H}_{2} \mathrm{O}_{2}$ sensitivity of $F$. tularensis LVS. We show that a LVS biovar that exhibits greater virulence in mice and engenders strong protective immunity possesses a single nucleotide polymorphism in the $f e o B$ gene that confers significantly higher resistance to $\mathrm{H}_{2} \mathrm{O}_{2}$ (due to lower iron acquisition) than other LVS biovars. In addition, we confirm that the modulation of iron uptake and the resulting sensitivity to $\mathrm{H}_{2} \mathrm{O}_{2}$ is not exclusive to $f e o B$, but can be extended to other iron homeostasis systems in the bacterium. We previously identified a $F$. tularensis gene involved in iron homeostasis from a mutagenesis screen to identify genes important for growth in human monocyte derived macrophages (MDMs) 
(Salomonsson et al., 2009). This earlier work identified the bacterioferritin $(b f r)$ gene as important for F. tularensis Schu $S 4$ growth in MDMs infected with pools of transposon mutants (Salomonsson et al., 2009). Specifically, we demonstrate that deletion of bacterioferritin ( $b f r$ ) increases sensitivity of the bacterium to $\mathrm{H}_{2} \mathrm{O}_{2}$, decreases virulence in vivo, and renders LVS poorly protective against challenge with virulent $F$. tularensis. Together, our findings connect the nutrient acquisition of LVS with its ability to provoke strong vaccine induced immunity.

\section{MATERIALS AND METHODS}

\section{Mutant Construction}

Deletion of $f e o B$ (FTL_0133) and bfr (FTL_0617) genes was achieved by homologous recombination using derivatives of the non-replicating plasmid pJC84. Primer sequences are shown in Table 1. Briefly, upstream and downstream flanking DNA was amplified via PCR, and amplicons were cloned into the multiple cloning site of pCR2.1 (Life Technologies). A spectinomycin resistance cassette was cloned into the AvrII site in the $3^{\prime}$ end of each upstream PCR fragment. The upstream-spectinomycin resistance fragment was removed by digestion with AscI, and cloned into the AscI site in the $5^{\prime}$ region of the downstream PCR plasmid. The entire upstream-spectinomycin resistancedownstream fragment was cloned into the BamHI site of the suicide plasmid pJC84. Finally, the spectinomycin resistance cassette was removed by digestion with AvrII and the plasmid was re-ligated. Plasmids were electroporated into LVS $(2.5 \mathrm{kV}$, $25 \mu \mathrm{F}$, and $600 \Omega$ ), and the bacteria were plated onto $\mathrm{MMH}$ agar with $50 \mu \mathrm{g} / \mathrm{mL}$ kanamycin after $2-3 \mathrm{~h}$ of outgrowth. Organisms were then grown overnight in broth lacking kanamycin, and

TABLE 1 | Primers used in this study.

5'feoB.up.asc1.bamh1-ggcgcgccggatccAGCATATCAAACACAAAGAAG AAGTATTG

3'feoB.up.avr2-cctaggAATCAGTITCAGGAGTTATAGTATAG

5'feoB.down.asc1.avr2-ggcgcgcccctaggAATTCTAATTTGAATATACAGCTTA

3'feoB.down.bgl2-agatctTTAGCATTTCACTAAGATTTGCACC

5'feoB.mut.check-AGATGATTGCTCAGTAATAACAGCAACTTTGC

3'feoB.mut.check-CATTAATAAGAATAGTATTCTCATITTAAAATACCTC

5'feoB.SNP-AGACTTGCGATATITTCAGTATTTGC

3'feoB.SNP-TTTACCGGCATATTCAAGTGCTGTGG

5'feoBc.kpn1-ggtaccATGAAATATGCTCTAGTTGGCAATCC

3'feoBc.sal1-gtcgacATATTTAAAGCTGTATATTCAAATTAG

5'feoAc.kpn1-ggtaccATGACTTATAACAAAAATGATAAATTTATTG

3'feoAc.sal1-gtcgacATATIIITCGTAAACGCATCTTATAGCCTC

5 'bferr.up.asc1.bamh1-ggcgcgccggatcc TITAGTGATACTIITGAGACA

ATTGTCCC

3'bferr.up.asc1.avr2 - ggcgcgcccctaggCATATTGTTACCTCCATTATTTAAAA CTCTAATC

5'bferr.down.asc1-ggcgcgccTAAAGGCTATTATCCTCGATGAGTITTCTTC

3'bferr.down.bamh1-ggatcCAAGTATTATCTGTAGTTACAATGGTGG

5'bfr.mut.check-ATATCATIITATTAAAATATCTAGGTTG

3'bfr.mut.check-ATAAATACTTAAGTCACTAAATATCTCG were plated onto $\mathrm{MMH}$ agar with $8 \%$ sucrose for $s a c B$-mediated counter-selection. Kanamycin sensitive colonies were screened by colony PCR to detect deletion of the $f e o B$ or $b f r$ gene. Primers were designed so that they flanked the coding sequence of each gene such that an amplicon would be produced regardless of genotype.

\section{Bacterial Strains and Growth Conditions}

Plasmids and bacterial strains used in this work at listed in Tables 2, 3, respectively. Three different biovars of F. tularensis subsp. holarctica LVS were used in this work. LVS obtained from Karen Elkins (FDA, Bethesda, MD) and routinely utilized at the University of Iowa, here called Iowa LVS (University of Iowa) and the RML LVS and ATCC LVS, (Rocky Mountain Laboratories) (Su et al., 2007). Bacteria were routinely cultured on modified Mueller-Hinton (supplemented with 1\% glucose, $0.025 \%$ ferric pyrophosphate, and $2 \%$ IsoVitaleX) agar or in broth, with $50 \mu \mathrm{g} / \mathrm{mL}$ of kanamycin or spectinomycin, as needed. MuellerHinton agar was also prepared without ferric pyrophosphate supplementation to assay for growth in lower iron conditions. For some experiments, bacteria were cultured in Chamberlain's defined medium with either $35 \mu \mathrm{M} \mathrm{FeSO}$ or $350 \mathrm{nM} \mathrm{FeSO}_{4}$, supplemented with antibiotics as needed. Agar plates were incubated at $37^{\circ} \mathrm{C}$ with humidity and $5 \% \mathrm{CO}_{2}$ while broth cultures were grown at $37^{\circ} \mathrm{C}$, shaken at $200 \mathrm{rpm}$.

\section{Murine Infection}

Eight to Ten week old female C57BL/6 mice were maintained on corncob bedding for 1 week prior to infection. Mice were intranasally infected with $25 \mu \mathrm{L}$ of various doses of $F$. tularensis LVS strains and mutant derivatives that had been resuspended in PBS. Inocula were calculated by measuring the $\mathrm{OD}_{600}$ value of mid- to late-log phase grown organisms, and were confirmed by serial dilution onto modified Mueller-Hinton agar and

\begin{tabular}{|c|c|c|}
\hline Plasmids & Description & Source \\
\hline pCR2.1TOPO & & Invitrogen \\
\hline pGEMT Easy & & Promega \\
\hline pBB103 & $\begin{array}{l}\text { Francisella-E. coli shuttle vector, } \\
\text { spect }^{R}\end{array}$ & \\
\hline pBB133 & $\begin{array}{l}\text { Francisella-E. coli shuttle vector } \\
\text { containing the } \mathrm{P}_{f s} / A^{-} \text {-lacZ reporter }\end{array}$ & $\begin{array}{l}\text { Buchan et al., } \\
2009\end{array}$ \\
\hline pJC84 & Francisella suicide vector & $\begin{array}{l}\text { Wehrly et al., } \\
2009\end{array}$ \\
\hline pJF103 & $\begin{array}{l}\text { Pgro-lowa LVS feoB in pBB103, } \\
\text { spect }^{R}\end{array}$ & This study \\
\hline pJF104 & Pgro-feoB D471Y in pBB103, spect ${ }^{R}$ & This study \\
\hline pJF109 & $\begin{array}{l}\text { Upstream and downstream feoB } \\
\text { homology arms in pJC84 }\end{array}$ & This study \\
\hline pJF114 & $\begin{array}{l}\text { Upstream and downstream bfr } \\
\text { homology arms in pJC84 }\end{array}$ & This study \\
\hline pJF118 & Pgro-feoA in pBB103 & This study \\
\hline pJF119 & lowa LVS feoB in pWKS30 & This study \\
\hline pJF120 & feoB D471Y in pWKS30 & This study \\
\hline
\end{tabular}


TABLE 3 | Strains.

\begin{tabular}{|c|c|c|}
\hline Strains & Description or genotype & Source \\
\hline $\begin{array}{l}\text { F. tularensis subsp. } \\
\text { holarctica RML LVS }\end{array}$ & & $\begin{array}{l}\text { RockyMountain } \\
\text { Laboratories }\end{array}$ \\
\hline $\begin{array}{l}\text { F. tularensis subsp. } \\
\text { holarctica lowa LVS }\end{array}$ & & Karen Elkins, FD \\
\hline $\begin{array}{l}\text { F. tularensis subsp. } \\
\text { holarctica ATCC LVS }\end{array}$ & & $\begin{array}{l}\text { RockyMountain } \\
\text { Laboratories }\end{array}$ \\
\hline JF200 & RML LVS carrying pJF103 & This study \\
\hline JF201 & RML LVS carrying pJF104 & This study \\
\hline JF209 & lowa LVS $\Delta f e o B$ & This study \\
\hline JF212 & JF209 carrying pJF103 & This study \\
\hline JF213 & JF209 carrying pJF104 & This study \\
\hline JF297 & RML LVS $\Delta b f r$ & This study \\
\hline JF305 & JF297 carrying pBB133 & This study \\
\hline E. coli Top 10 & $\begin{array}{l}\text { F- mcrA } \Delta(\text { mrr-hsdRMS-mcrBC) } \\
\text { \$80lacZ } \Delta \text { M15 } \Delta \text { lacX74 recA1 } \\
\text { araD139 } \Delta \text { (araleu)7697 galU } \\
\text { galK rpsL (StrR) endA1 nupG }\end{array}$ & Invitrogen \\
\hline E. coli $\mathrm{H} 1771$ & $\begin{array}{l}\text { MC4100 aroB feoB7 fhuF::plac } \\
\text { Mu }\end{array}$ & $\begin{array}{l}\text { Kammler et al., } \\
1993\end{array}$ \\
\hline JF280 & $\begin{array}{l}\text { pJF118 and pJF119 in E. coli } \\
\mathrm{H} 771\end{array}$ & This study \\
\hline JF281 & $\begin{array}{l}\text { pJF118 and pJF120 in E. coli } \\
\mathrm{H} 771\end{array}$ & This study \\
\hline
\end{tabular}

enumeration after $2-3$ days growth at $37^{\circ} \mathrm{C}$ with humidity, $5 \%$ $\mathrm{CO}_{2}$. Moribund animals (defined as having lost $25 \%$ of the initial body weight) were sacrificed in accordance with the protocol approved by the University of Iowa Institutional Animal Care and Use Committee.

\section{Genome Sequencing}

Genomic DNA from RML LVS and ATCC LVS was prepared from overnight cultures using DNeasy tissue kit (Qiagen) according to the instructions of the manufacturer. DNA was sequenced using an Illumina HiSeq2500 platform by the Institute for Genomic Medicine Genomic Center (University of California, San Diego, San Diego, CA), resulting in $\sim 7$ million reads per sample. Reads were trimmed for adapter sequence and trimmed and filtered for low quality bases using the FASTXToolkit (Hannon Lab, CSHL). Resulting reads were mapped to the LVS genome (NC_007880) using Bowtie2 (Ashkenazy et al., 2010). Analysis of genetic modifications, e.g., SNPs and gene deletions was performed using GATK comparing both RML LVS and ATCC LVS genomes to each other and the annotated LVS genome (Landau et al., 2005).

\section{Iron-Regulated $\beta$-Galactosidase Reporter Activity}

To assess iron uptake-regulated gene expression, Miller assays were performed with Iowa LVS, RML LVS, and ATCC LVS carrying the $f$ slA promoter-lacZ fusion on the pBB103 plasmid (Troxell and Hassan, 2013). Bacteria were grown in Chamberlain's defined medium (supplemented with $50 \mu \mathrm{g} / \mathrm{mL}$ spectinomycin) overnight, and $\beta$-galactosidase activity was assayed using the standard Miller assay (Sullivan et al., 2006).

\section{FeoB Function in a Heterologous Reporter System}

The coding sequence of $f e o B$ was PCR-amplified from both Iowa and RML LVS using the high fidelity Phusion polymerase (New England Biolabs). Sanger sequencing was performed to confirm that the SNP observed in the RML LVS background was present. The feoA coding sequence was cloned into the KpnI/SalI sites of a derivative of pTrc99a, immediately downstream of the groE promoter. The entire $\mathrm{P}_{g r o E}-f e o A$ fragment was removed by digestion with BamHI and SalI, and ligated into the same sites in pBB103. Both this plasmid and the pWKS30 containing $f e o B$ were transformed into the E. coli H1771 strain (MC4100 aroB feoB7 fhuF::plac $\mathrm{Mu}$ ). Control strains were also generated that carried only one of the plasmids. Miller assays were performed to measure $\mathrm{FeoB} \mathrm{Fe}^{2+}$ import activity via the readout of Fur repression of $f h u F:$ placZ. All strains were grown in LB supplemented with $50 \mu \mathrm{g} / \mathrm{mL}$ of spectinomycin and $100 \mu \mathrm{g} / \mathrm{mL}$ of ampicillin, as necessary.

\section{Hydrogen Peroxide Sensitivity}

To measure resistance to $\mathrm{H}_{2} \mathrm{O}_{2}$-mediated killing, bacteria were grown in modified Mueller-Hinton broth and mid- to late-log phase organisms were pelleted at 13,200 RPM for $5 \mathrm{~min}$, washed in PBS, and $\sim 10^{6}$ CFU were resuspended in $200 \mu \mathrm{L}$ PBS with or without $100 \mu \mathrm{M}$ hydrogen peroxide $\left(\mathrm{H}_{2} \mathrm{O}_{2}\right)$ in a 96-well dish. The samples were incubated at $37^{\circ} \mathrm{C}$ with humidity and $5 \% \mathrm{CO}_{2}$ for $1 \mathrm{~h}$. The culture from each well was then serially diluted in sterile PBS, plated onto modified Mueller-Hinton agar (with antibiotic when necessary) and the number of surviving organisms for each strain was enumerated after 2-3 days.

\section{In Vitro Infections}

LVS infections of bone marrow derived macrophages (BMM) were performed as previously described (Su et al., 2007). Briefly, BMM were differentiated from femurs of $\mathrm{C} 57 \mathrm{Bl} / 6$ mice over the course of 7 days in complete DMEM (cDMEM, DMEM supplemented with $10 \%$ heat-inactivated fetal calf serum [FCS], $0.2 \mathrm{mM}$ L-glutamine, $1 \mathrm{mM}$ HEPES, and $0.1 \mathrm{mM}$ non-essential amino acids [NEAA], all from Life Technologies) supplemented with M-CSF (Peprotech). Immediately prior to infection medium was removed and reserved for addition after infection. The indicated bacterial strains were added at a MOI of 50 and coincubated with BMM for $90 \mathrm{~min}$. Bacteria were then removed and fresh medium containing $50 \mu \mathrm{g} / \mathrm{ml}$ of gentamicin was added to kill extracellular organisms. After $45 \mathrm{~min}$, BMM were washed extensively with D-PBS (Life Technologies) and the reserved medium was returned to the wells. IFN- $\gamma$ (Peprotech) was then added to the indicated groups at a final concentration of $10 \mathrm{U} / \mathrm{ml}$. At the indicated time points, medium was collected and assessed for cytokines as described below. BMM were washed 3 times with D-PBS followed by lysis with water. Lysates were serially diluted and plated on to MMH agar for enumeration of viable organisms. 


\section{Statistics}

All statistics were calculated with Graphpad Prism software (San Diego, CA). To compare $ß$-galactosidase activity across multiple strains we used one-way ANOVA with Dunnett's multiple comparisons test. For comparisons of multiple strains across multiple conditions, we utilized two-way ANOVA with either Sidak's or Tukey's multiple comparisons tests. When applicable, an unpaired two-tailed $t$-test was used. The $\mathrm{LD}_{50}$ values were calculated using the method of Reed and Muench. To compare survival after F. tularensis SchuS4 challenge, a log-rank sum test was utilized. The error bars in the presented data represent the standard error of the mean. A $p<0.0001$ is represented by **** $p$ $<0.01$ is represented by ${ }^{* *} p<0.05$ is represented by *, and "ns" indicates "not significant."

\section{RESULTS}

\section{Genome Sequencing of $F$. tularensis LVS Strain}

It was recently reported that there are differences in virulence and vaccine efficacy between LVS isolates (Deng et al., 2006; Su et al., 2007). Specifically, a low passage strain, termed RML LVS, had increased virulence in $\mathrm{C} 57 \mathrm{Bl} / 6$ mice but also engendered complete protection against challenge with virulent $F$. tularensis subsp tularensis. In contrast, LVS strain ATCC29684 (ATCC LVS) had lower virulence in $\mathrm{C} 57 \mathrm{Bl} / 6$ mice and failed to protect animals from lethality following $F$. tularensis challenge. The specific genetic changes that could account for the dramatic in vivo differences between these two strains were not identified. Therefore, in this work we have sequenced the genomes of these two LVS biovars to identify and characterize these genetic differences. Surprisingly, we detected very few differences in the sequences between RML LVS and ATCC LVS. We detected a 93 base pair deletion in the gene encoding a Dyp-type peroxidase that has been previously characterized (Binesse et al., 2015). Additionally, we detected a single nucleotide polymorphism in the $f e o B$ gene encoded by RML LVS. The $C$ to A substitution leads to an aspartate to tyrosine mutation in the coding sequence of $f e o B$. The D471Y mutation maps to a cytoplasmic loop of the FeoB protein, adjacent to a highly conserved glycine residue that is predicted to be functionally important by the ConSurf program (Kammler et al., 1993; Buchan et al., 2008). Although the genome of the lab stock of Iowa LVS from the Jones lab has not been completely sequenced, the strain was also included in the experiments described here. Sanger sequencing confirmed that the Iowa LVS $f e o B$ encodes an aspartate at residue 471, similar to the published LVS genome. FeoB is an inner membrane protein that imports ferrous iron into the bacterial cytoplasm and has previously been linked to virulence as one of two major iron uptake pathways in LVS (Schulert et al., 2009; Thomas-Charles et al., 2013; Perez and Ramakrishnan, 2014). The substitution of a large, hydrophobic tyrosine for an aspartate at residue 471 led us to hypothesize that the FeoB protein encoded by the RML LVS allele imports $\mathrm{Fe}^{2+}$ poorly, or not at all. We determined if differences in iron acquisition could account for the varied virulence and vaccine efficacy of RML LVS vs. ATCC
LVS. Since iron plays an integral role in host-induced oxidative stress, it is likely that controlling intracellular iron levels is a significant component of the virulence strategy of $F$. tularensis. Furthermore, sensitivity to oxidative stress may contribute to the vaccine efficacy of LVS given that the ATCC biovar of LVS has an aspartate at residue 471 in $\mathrm{FeoB}$ and the 93 base pair $d y p$ deletion, and is both relatively attenuated in murine infections and stimulates a weak immune response against a challenge with virulent F. tularensis Schu S4 (Griffin et al., 2015).

\section{RML LVS Displays Phenotype Consistent With Lower Intracellular $\mathrm{Fe}^{2+}$ Than the ATCC LVS or lowa LVS}

Our first set of experiments was designed to test the hypothesis that RML LVS has less intracellular iron than either the Iowa LVS or the ATCC LVS. Strains were grown overnight in standard modified Mueller-Hinton (MMH) broth, serially diluted in PBS, and ten-fold dilutions of liquid bacterial cultures were spotted onto MMH agar with varying concentrations of iron (Figure 1A). Growth was identical among each strain when spotted onto the control agar containing the typical concentration of iron ( $0.0025 \%$ ferric pyrophosphate) routinely used for propagation of F. tularensis. When the iron concentration was reduced by $50 \%$, both the Iowa LVS and ATCC LVS had growth patterns similar to that observed on the control plate, however, RML LVS exhibited growth restriction at this concentration of iron. Fewer isolated colonies were observed and lawn growth was less luxurious when compared to the Iowa and ATCC LVS. On MMH agar lacking added iron, all three strains exhibited growth restriction, however the reduced growth phenotype of RML LVS was exacerbated, with extremely poor growth even at the lowest dilution plated. Iowa and ATCC LVS both grew as lawns at these dilutions, indicating that they were able to scavenge sufficient iron from the agar plate environment, while RML LVS could not. The decrease in RML LVS growth was approximately two orders of magnitude greater than that observed for either the Iowa or ATCC LVS, consistent with the hypothesis that RML LVS has less intracellular iron under conditions of iron limitation. We interpreted these results to mean that RML LVS had inherently lower levels of intracellular iron and that when growing the strain on agar with less iron, the amount that RML LVS could acquire for growth was limiting, thus the strain grew poorly relative to the Iowa LVS or ATCC LVS.

To confirm that the mutation carried in $f e o B$ by RML LVS had a direct consequence on iron levels, we performed experiments designed to give a semi-quantitative measure of intracellular iron levels. To avoid toxicity associated with the Fenton reaction that include lipid peroxidation, DNA damage, and poisoning of the iron-sulfur cluster enzymes of essential metabolic pathways, bacteria have evolved regulatory mechanisms to maintain iron homeostasis. One such example is the Fur system (Troxell and Hassan, 2013). The Fur transcriptional repressor is an allosteric regulator that uses $\mathrm{Fe}^{2+}$ as a co-repressor; when iron levels are sufficient, the Fur- $\mathrm{Fe}^{2+}$ complex binds to Fur Box sequence motifs in the promoters of iron uptake genes to mediate their repression. When iron is limiting, 


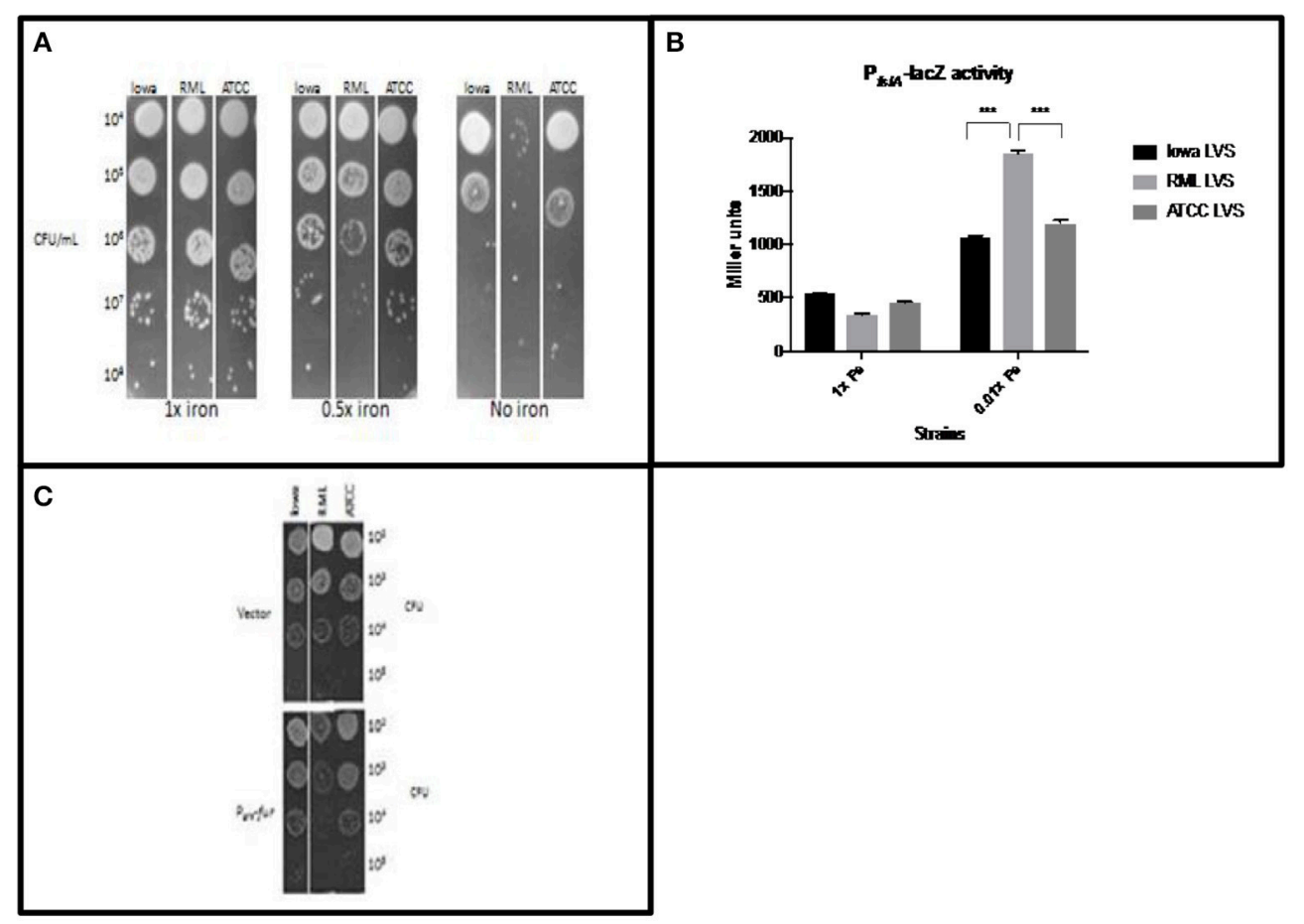

FIGURE 1 | RML LVS has relatively less intracellular iron than other LVS biovars. (A) Bacteria were grown overnight in standard MMH broth, serially diluted in sterile PBS and plated for enumeration on $\mathrm{MMH}$ agar with $0.025 \%, 0.0125 \%$, or no added Fe ${ }^{3+} \mathrm{PP}_{\mathrm{j}}$. Data from a representative experiment is shown. (B) Miller assay for each LVS carrying a plasmid with the Fur regulated $\mathrm{P}_{f s / A}$-lacZ reporter. Bacteria were grown overnight in Chamberlain's defined medium with $35 \mu \mathrm{M}$ (high iron) or $350 \mathrm{nM}$ (low iron) $\mathrm{FeSO}_{4}$. Two-way ANOVA with Sidak's multiple comparisons was used to test for significance. Shown is a representative Miller assay from replicate experiments. (C) Strains overexpressing fur were grown in $\mathrm{MMH}$ broth overnight, serially diluted into sterile PBS and plated on standard MMH agar. Shown are representative data from two similarly designed experiments.

less $\mathrm{Fe}^{2+}$ is bound to Fur, decreasing its ability to bind DNA. This leads to de-repression of iron uptake genes. Since several studies have demonstrated that Francisella has a Fur regulatory system that functions similarly to other organisms (Deng et al., 2006; Sullivan et al., 2006; Buchan et al., 2008; Ramakrishnan et al., 2008), we were able to design experiments to indirectly measure $F$. tularensis LVS intracellular iron levels. A plasmid carrying a Fur-regulated $\mathrm{P}_{f_{s} l A}$-lac $Z$ construct was introduced into the RML LVS, Iowa LVS, and ATCC LVS. Iron limitation is known to relieve Fur repression at the $f_{s} l A$

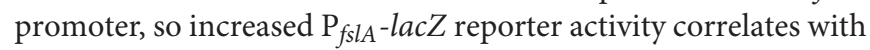
decreasing concentrations of intracellular $\mathrm{Fe}^{2+}$ (Buchan et al., 2009). Each LVS strain was grown in Chamberlain's defined medium (CDM) with $35 \mu \mathrm{M}$ (high iron) or $350 \mathrm{nM}$ (low iron) $\mathrm{FeSO}_{4}$, and $\beta$-galactosidase activity was measured after overnight growth (Figure 1B). As predicted, iron starvation induced high expression of $\mathrm{P}_{f s l A}$-lac $Z$ in all three strains; however, the lac $Z$ reporter expression was approximately 2-fold higher in RML LVS than that observed for Iowa LVS or ATCC LVS. The higher activity of the $\mathrm{P}_{f_{s l} \text { A }}$-lacZ construct in the RML LVS is consistent with the hypothesis that this strain has lower levels of intracellular $\mathrm{Fe}^{2+}$ when iron is limiting in the growth medium.
To assess intracellular iron levels in RML LVS by another method, we reduced the ability of each strain to import iron by overexpression of Fur (which downregulates genes encoding iron uptake systems). The F. tularensis fur gene was placed under the control of the groE promoter, and the construct was introduced into each strain. Bacteria were grown in $\mathrm{MMH}$ broth with standard iron concentrations overnight (no differences in growth rate were observed between the two strains in iron replete conditions), serially diluted in PBS and plated onto standard MMH agar (Figure 1C). Iowa LVS and ATCC LVS, when overexpressing Fur, exhibited no significant difference in colony growth compared to vector controls, indicating that intracellular pools of iron were still sufficiently high to support normal growth, even with Fur overexpression. In contrast, RML LVS had much smaller colony size and poor lawn formation. We interpret these results to indicate that Fur overexpression in RML LVS had a greater impact on growth because Fur repression of iron uptake genes decreased the intracellular $\mathrm{Fe}^{2+}$ concentration to levels such that the strain could not grow normally, even though sufficient iron was present in the agar media. These results are consistent with the hypothesis that intracellular $\mathrm{Fe}^{2+}$ pools of RML LVS are lower than in Iowa LVS or ATCC LVS and can be manipulated to become limiting 
for growth in conditions that are not limiting for the other two strains.

\section{FeoB D471Y Does Not Complement E. coli $\Delta$ feoB fhuF:: $\lambda$ plac}

The data demonstrating that RML LVS has lower intracellular pools of iron than either Iowa LVS or ATCC LVS suggest that the single nucleotide change in the $f e o B$ gene of the RML strain encodes a protein with significantly reduced $\mathrm{Fe}^{2+}$ import activity. To assess the ability of the FeoB D $471 Y$ protein to transport iron, we made use of a well-characterized $E$. coli iron reporter strain that lacks feoB (Kammler et al., 1993; Weaver et al., 2013). This strain, E. coli $\mathrm{H} 1771$, has a chromosomal transcriptional lacZ reporter in the Fur-regulated gene $f h u F$ which can be used to report the relative intracellular iron concentrations within the strain. When $\mathrm{Fe}^{2+}$ levels are high, expression of the fhuF::lacZ reporter is low and when $\mathrm{Fe}^{2+}$ levels are low, high $\beta$-galactosidase activity is observed. The $f e o B$ alleles from Iowa LVS and RML LVS (D471Y) were introduced into E. coli H1771 on the low copy number plasmid pWKS30, and Miller assays were performed (Figure 2). Initial experiments supplying only $f e o B$ (either allele) showed no repression of $f h u F:$ :lac $Z$, indicating that FeoB alone is not sufficient for $\mathrm{Fe}^{2+}$ import in this reporter system (data not shown). In most bacterial species encoding a feo system, the small $f e o A$ gene is encoded either in an operon with $f e o B$ or elsewhere on the chromosome and the encoded protein is required to stimulate FeoB Fe ${ }^{2+}$ import activity (Cartron et al., 2006; Lau et al., 2013; Weaver et al., 2013). When the Francisella feoA was supplied on pBB103 in concert with $f e o B$ (Iowa/ATCC allele) on pWKS30, significant repression of the fhuF-lacZ reporter was observed ( $\sim 80 \%$ reduction in expression relative to the empty vector control), indicating that $\mathrm{Fe}^{2+}$ import was significantly increased. In contrast, complementation of $\mathrm{H} 1771$ with $f e o A$ and feoB D471Y (RML allele) failed to repress fhuF::lacZ. This result is consistent with the hypothesis that the FeoB D471Y protein from the RML LVS is significantly impaired in its ability to import $\mathrm{Fe}^{2+}$.

\section{The feoB d471Y Allele Is Associated With Resistance to $\mathrm{H}_{2} \mathrm{O}_{2}$}

Since we have provided several lines of evidence consistent with the hypothesis that RML LVS has less intracellular $\mathrm{Fe}^{2+}$ than either the Iowa or ATCC LVS strains and Lindgren et al. demonstrated that strains with lower intracellular iron concentrations are more resistant to $\mathrm{H}_{2} \mathrm{O}_{2}$, we hypothesized that RML LVS would be more resistant to killing by $\mathrm{H}_{2} \mathrm{O}_{2}$ than Iowa LVS or ATCC LVS. Bacteria were exposed to PBS alone or PBS with $100 \mu \mathrm{M} \mathrm{H}_{2} \mathrm{O}_{2}$ in a 96-well dish for $1 \mathrm{~h}$ at $37^{\circ} \mathrm{C}$ with $5 \% \mathrm{CO}_{2}$ and humidity. Following incubation, the bacteria were serially diluted in PBS and plated onto $\mathrm{MMH}$ agar (Figure 3A). The RML LVS was typically ten-fold more resistant to $\mathrm{H}_{2} \mathrm{O}_{2}$ than the Iowa strain, and approximately 100 -fold to 1,000-fold more resistant than ATCC strain. The genome of ATCC encodes a Dyp peroxidase with a 93-base pair deletion, and it has been reported that this deletion can contribute to increased sensitivity to $\mathrm{H}_{2} \mathrm{O}_{2}$; an Iowa LVS $\Delta d y p$ mutant matches the $\mathrm{H}_{2} \mathrm{O}_{2}$ sensitivity of ATCC

\section{E. coli $\triangle$ feoB fhuF::Aplac Mu $\beta$-galactosidase activity}

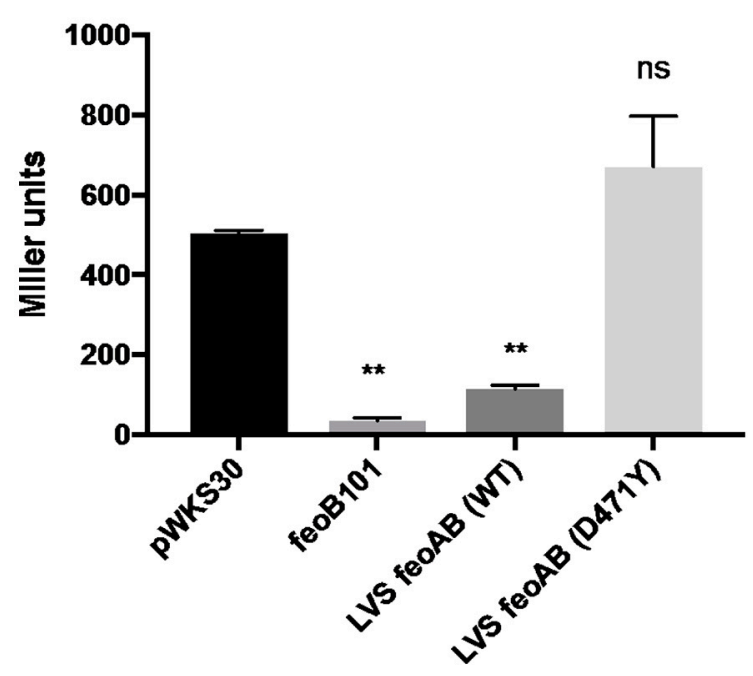

FIGURE 2 | The RML LVS feoB allele is non-functional in a heterologous iron reporter system. The coding sequence of each $f e o B$ allele was cloned into the low copy plasmid pWKS30 and introduced to E. coli H1771. The coding sequence of feoA was fused to the groE promoter in a derivative of plasmid pBB103 and supplied to $E$. coli $\mathrm{H} 1771$ in tandem with the feoB expression plasmids, and Miller assays were performed as described in the Materials and Methods. A one-way ANOVA with Dunnett's multiple comparisons test was used to test for significance against the control pWKS30 plasmid. Significance was set at $p<0.05$. ${ }^{\star}$ Indicates a significant difference between the labeled samples and pWKS30. Shown is a representative experiment from three replicates.

LVS (data not shown) and is in agreement with the data from Binesse et al. (2015).

Given the increased sensitivity of Iowa LVS to $\mathrm{H}_{2} \mathrm{O}_{2}$, and the functional complementation of an $E$. coli feoB mutant by the Iowa $f e o B$ allele, we next tested if the RML LVS could be sensitized to $\mathrm{H}_{2} \mathrm{O}_{2}$ by increasing the intracellular $\mathrm{Fe}^{2+}$ pool via overexpression of a functional $f e o B$. This was achieved by transforming the strain with a plasmid carrying constructs $\mathrm{P}_{\text {gro }}{ }^{-}$ feoB (Iowa/ATCC allele-high $\mathrm{Fe}^{2+}$ transport) and $\mathrm{P}_{\text {gro }}-f e o B$ (D471Y; RML allele-low $\mathrm{Fe}^{2+}$ transport) which both overexpress feoB. $\mathrm{H}_{2} \mathrm{O}_{2}$ sensitivity assays were performed as described previously, except that a 30 min $\mathrm{H}_{2} \mathrm{O}_{2}$ exposure time point was also included in case overexpression of the $f e o B$ genes led to substantially faster killing by hydrogen peroxide (Figure 3B). Nearly one log of killing was observed at $30 \mathrm{~min}$ for the RML + $\mathrm{P}_{\text {gro }}-f e o B$ (Iowa/ATCC), while RML $+\mathrm{P}_{\text {gro }}-f e o B(\mathrm{D} 471 \mathrm{Y})$ only had a two-fold reduction in viability. The effect was significantly more pronounced at the $1 \mathrm{~h}$ time point, with no viable bacteria recovered at the level of detection $\left(10^{2} \mathrm{CFU}\right)$ from $\mathrm{RML}+\mathrm{P}_{\text {gro }}{ }^{-}$ feoB (Iowa/ATCC). In contrast, over $10^{4} \mathrm{CFU}$ were recovered at $1 \mathrm{~h}$ from the $\mathrm{RML}+\mathrm{P}_{\text {gro }}-\mathrm{feoB}(\mathrm{D} 471 \mathrm{Y})$. These data demonstrate that the single nucleotide change in the $f e o B$ gene (D471Y0 encoded in the RML LVS genome results in significantly higher resistance to $\mathrm{H}_{2} \mathrm{O}_{2}$ compared to the $f e o B$ allele in either Iowa LVS or ATCC LVS. 


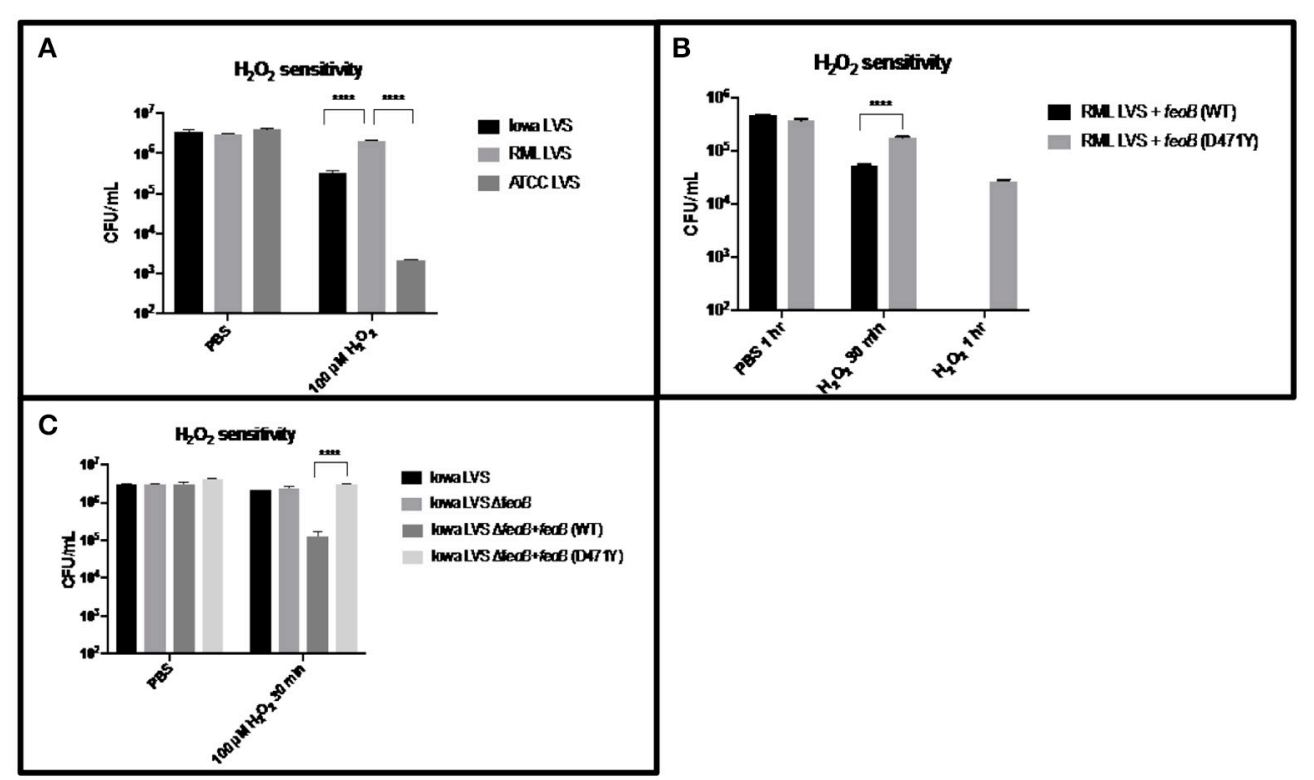

FIGURE 3 | RML LVS is more resistant to $\mathrm{H}_{2} \mathrm{O}_{2}$ as a result of the point mutation in its feoB. (A) Strains were grown overnight in $\mathrm{MMH}$ broth, resuspended to $\sim 10^{6}$ CFU in PBS and exposed to $100 \mu \mathrm{M} \mathrm{H}_{2} \mathrm{O}_{2}$ for $1 \mathrm{~h}$ at $37^{\circ} \mathrm{C}$. Samples were serially diluted in sterile PBS and plated in triplicate. Shown is the CFU/ml in PBS alone and $\mathrm{PBS}+\mathrm{H}_{2} \mathrm{O}_{2}$ from a representative experiment. A two-way ANOVA with Tukey's multiple comparisons test was used to test for significance. Shown is a representative of 3 replicates. (B) Each $f e o B$ allele was constitutively expressed from the gro $E$ promoter in a derivative of the multi-copy plasmid pBB103. These plasmids were introduced into the RML LVS and $\mathrm{H}_{2} \mathrm{O}_{2}$ sensitivity assays were performed as before, with the addition of a 30-min time point. Shown is the CFU/ml in PBS alone and $\mathrm{PBS}+\mathrm{H}_{2} \mathrm{O}_{2}$ from a representative experiment. A two-way ANOVA with Sidak's multiple comparison test was used to test for significance. Shown is a representative of two replicates. (C) An lowa LVS $\triangle$ feoB mutant was complemented with each feoB allele in trans, and $\mathrm{H}_{2} \mathrm{O}_{2}$ sensitivity assays were performed as before. A two-way ANOVA with Tukey's multiple comparisons test was used to test for significance. Significance was set at $p<0.05 .{ }^{\star \star \star \star} \mid$ Indicates a significant difference between the two samples bracketed. Shown is a representative of two replicates.

To confirm that the $\mathrm{H}_{2} \mathrm{O}_{2}$ resistance phenotype conferred by $f e o B$ D471Y is not unique to the RML genetic background, we constructed a $\triangle f e o B$ mutant in the Iowa LVS background and complemented it with the $\mathrm{P}_{g r o}-f e o B$ constructs. The Iowa LVS strain background was chosen over the ATCC LVS background, as the latter strain was approximately 100 -fold more sensitive to $\mathrm{H}_{2} \mathrm{O}_{2}$ than the RML LVS due to the 93 base pair deletion in the $d y p$ peroxidase gene, while RML LVS and Iowa LVS both encode a full length $d y p . \mathrm{H}_{2} \mathrm{O}_{2}$ sensitivity assays were performed as described previously and, consistent with results in the RML strain background, overexpression of $f e o B$ D471Y mediated significant resistance to $\mathrm{H}_{2} \mathrm{O}_{2}$ in the Iowa LVS $\triangle f e o B$ mutant, relative to that when the Iowa/ATCC $f e o B$ was overexpressed (Figure 3C).

\section{Bacterioferritin Protects Against Hydrogen Peroxide}

To assess the role of the $b f r$ gene in $F$. tularensis iron homeostasis and oxidative stress resistance, a $\Delta b f r$ strain was constructed in the RML LVS and $\mathrm{P}_{f s l A}$-lacZ assays, as well as $\mathrm{H}_{2} \mathrm{O}_{2}$ sensitivity assays were performed as described above. No growth differences were observed in the $\Delta b f r$ mutant compared to the wild type strain. The RML LVS $\Delta b f r$ strain exhibited a modest decrease $(\sim 20 \%)$ in $\mathrm{P}_{\text {fslA }}$-lac $Z$ reporter activity relative to the parent strain (Figure 4A), while in the $\mathrm{H}_{2} \mathrm{O}_{2}$ sensitivity assay, as before, the RML LVS exhibited relatively high level resistance to $\mathrm{H}_{2} \mathrm{O}_{2}$.
The $\Delta b f r$ mutant had $\sim 10$-fold fewer viable organisms, similar to Iowa LVS (Figure 4B). A possible explanation for these results is that in the absence of the Bfr iron storage protein, intracellular ferrous iron levels are elevated relative to the parent RML LVS, which may lead to increased ferrous iron available for the Fenton reaction in the presence of $\mathrm{H}_{2} \mathrm{O}_{2}$ and the increased toxicity observed in the assay. Consistent with this, $\mathrm{P}_{\text {fslA }}$-lac $Z$ activity was modestly reduced $(\sim 20 \%)$ in the RML LVS $\Delta b f r$ mutant (Figure 4B). As a result, we conclude that bacterioferritin contributes to protection against oxidative stress, consistent with bacterioferritin function in Neisseria gonorrhea and Mycobacterium tuberculosis.

\section{Expression of the lowa/ATCC LVS feoB Allele Is Deleterious in IFN- $\gamma$-Stimulated Macrophages}

One mechanisms by which IFN- $\gamma$ can control intracellular bacteria is to provoke production of reactive nitrogen and reactive oxygen species. It has been reported that IFN- $\gamma$ mediated control of Francisella replication can be independent of RNS and ROS (Edwards et al., 2010). However, this may be due to its ability to limit toxicity of ROS by restricting iron. Therefore, we hypothesized that strains of LVS that were unable to control iron acquisition similarly to wild type RML LVS would be more sensitive to host cell IFN- $\gamma$ mediated killing. We first compared RML LVS, ATCC LVS, RML LVS $\triangle f e o B$ and RML 


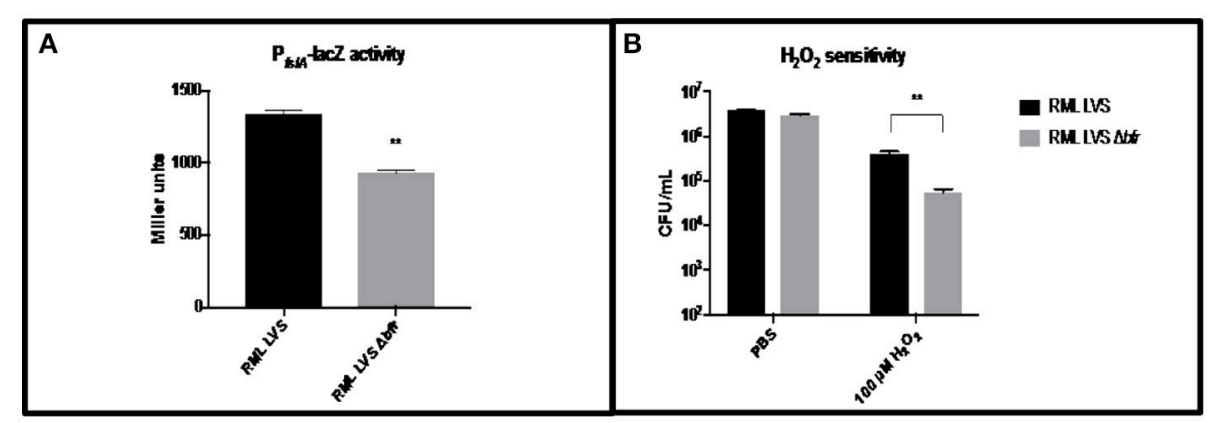

FIGURE 4 | Iron related phenotypes of RML LVS $\triangle$ bfr. (A) $P_{f S / A}$-lacZ reporter activity is decreased in the RML LVS relative to wild type. Welch's $t$-test was used to test for significance. Shown is a representative of five replicates. (B) The RML LVS $\triangle b f r$ is more sensitive to $\mathrm{H}_{2} \mathrm{O}_{2}$ killing than the wild type. A two-way ANOVA with Sidak's multiple comparisons test was used to test for significance. Significance was set at $p<0.05$. **Indicates a significant difference between the samples indicated. Shown is a representative of four replicates.

LVS overexpressing RML $f e o B$ or Iowa/ATCC $f e o B$. In agreement with our hypothesis RML LVS was the least sensitive to IFN$\gamma$ mediated killing among the strains tested (Figures 5A,B). Deletion of the $f e o B$ gene rendered the bacteria significantly more sensitive to IFN- $\gamma$ mediated killing (Figures $5 \mathbf{A}, \mathbf{B}$ ). However, resistance to IFN- $\gamma$ was partially restored in bacteria complemented with RML feoB. Complementation with ATCC $f e o B$ did not significantly change RML LVS sensitivity to IFN- $\gamma$ mediated killing. We next tested the ability of RML LVS $\Delta b f r$ to resist IFN $-\gamma$ mediated killing. Similar to RML LVS $\Delta f e o B$, RML LVS $\Delta b f r$ was significantly more sensitive to IFN- $\gamma$ mediated killing compared to wild type controls (Figures 5C,D). Together these data suggest that alterations in ferrous iron homeostasis confer sensitivity to IFN- $\gamma$ mediated control of intracellular replication of LVS.

\section{RML LVS $\Delta b f r$ Is Attenuated in Murine Infection and Fails to Protect Against F. tularensis SchuS4}

As strains with increased sensitivity to $\mathrm{H}_{2} \mathrm{O}_{2}$ are associated with IFN $\gamma$-mediated killing in BMMs, we sought to determine if strains with increased sensitivity to $\mathrm{H}_{2} \mathrm{O}_{2}$ in vitro might also be attenuated in a mouse model of respiratory tularemia. Disappointingly, several efforts to assess virulence of Iowa LVS, its isogenic $\triangle f e o B$ mutant and complemented derivatives gave inconclusive results, likely due to plasmid instability in the LVS strains in the absence of antibiotic selection in vivo. Thus, we pursued an alternative approach to assay for the role of altered ferrous iron homeostasis in vivo and the ability of RML LVS to provide protection against a challenge with $F$. tularensis SchuS4. To this end, we used the $\mathrm{H}_{2} \mathrm{O}_{2}$ sensitive RML LVS $\Delta b f r$ mutant in murine intranasal infections. RML LVS was previously reported to have a $\mathrm{LD}_{50}$ of $174 \mathrm{CFU}$ in C57BL6 mice (Griffin et al., 2015). We confirmed this value by performing a similar infection experiment and calculated a $\mathrm{LD}_{50}$ value for RML LVS in C57BL/6 mice of 195 CFU (Figure 6A). To test if bacterioferritin contributed to the virulence of the F. tularensis RML LVS, three groups of mice ( $n=5$ per group) were intranasally infected with $215 \mathrm{CFU}, 2.15 \times 10^{3}$, or $2.15 \times 10^{4} \mathrm{CFU}$ of the RML LVS
$\Delta b f r$ mutant, and their weight and health status was followed daily over the course of the experiment. All mice that received 215 CFU survived (average weight loss of $\sim 12$ percent) while forty percent of the mice survived infection with $2 \times 10^{3} \mathrm{CFU}$ (average weight loss of $\sim 22 \%$ ); none survived $2 \times 10^{4} \mathrm{CFU}$. Importantly, it took $>2,000 \mathrm{CFU}$ of the RML LVS $\Delta b f r$ strain to yield survival curves and weight losses in mice that approximated that observed for mice infected with only 133 CFU of RML LVS, a $>15$-fold difference in inoculum (Figures 6B,C). The estimated $\mathrm{LD}_{50}$ of the RML LVS $\Delta b f r$ mutant was $1,464 \mathrm{CFU}$. These results demonstrate that the bacterioferritin protein contributes to the virulence of RML LVS in mice; however, future work is needed to determine if this is a consequence of the $\mathrm{H}_{2} \mathrm{O}_{2}$ sensitivity of this mutant.

Wild type RML LVS uniformly protects C57Bl/6 mice against challenge with virulent Francisella whereas ATCC LVS does not (Griffin et al., 2015). We have established that an important difference between RML LVS and ATCC LVS is their acquisition of iron and subsequent sensitivity to ROS that is inversely correlated with protective efficacy. Since mutation of $b f r$ in RML LVS led to a decrease in $\mathrm{P}_{f s l A}$-lac $Z$ reporter activity, increased sensitivity to $\mathrm{H}_{2} \mathrm{O}_{2}$, and decreased virulence we hypothesized that vaccination with RML LVS $\Delta b f r$ would provide poor protection against challenge with virulent $F$. tularensis SchuS4. Accordingly, 6 weeks after infection with the RML LVS $\Delta b f r$ mutant, we challenged all animals that survived with $F$. tularensis SchuS4 (Figure 7). As a control, mice that had been intranasally infected with $121 \mathrm{CFU}$ of RML LVS $(n=3)$ were also challenged with $25 \mathrm{CFU}$ of $F$. tularensis SchuS4. As expected all mice that had previously been infected with RML LVS survived SchuS4 challenge. In contrast, none of the mice previously infected with RML LVS $\Delta b f r$ strain were protected from the SchuS4 infection and all mice died within 8 days of challenge. Thus, while prior infection with RML LVS $\Delta b f r$ did extend the mean time to death, it provoked incomplete protection against subsequent infection with fully virulent SchuS4. Together this suggests that Francisella iron homeostasis and its interplay with host elements that control bacterial replication are important factors that contribute to both survival of primary infection and induction of adaptive immune responses in vivo. 


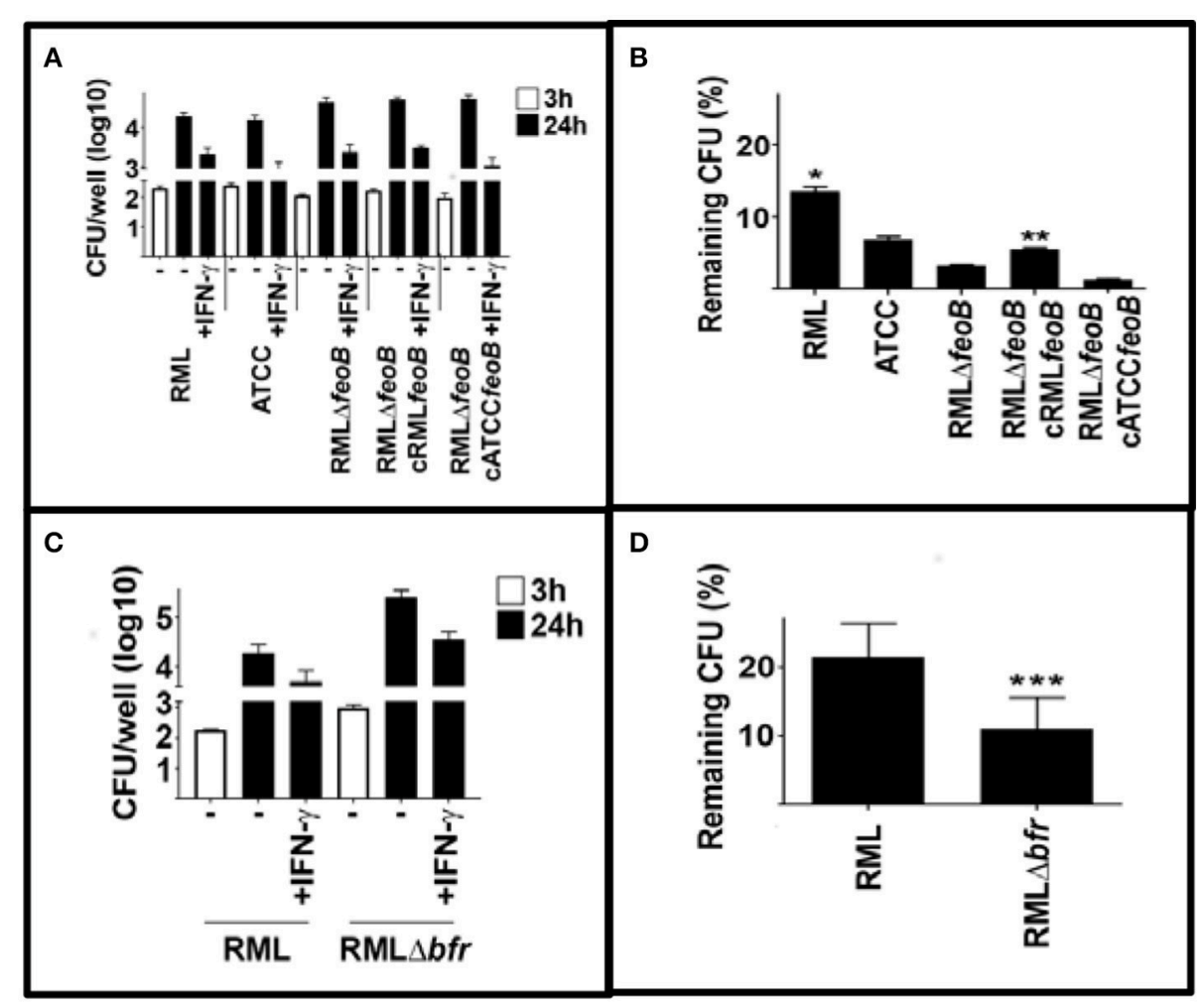

FIGURE 5 | Mutations in iron acquisition enhance sensitivity of LVS to IFN- $\gamma$ mediated killing. BMM were infected with the indicated strains of LVS and were treated with IFN- $\gamma(10 \mathrm{U} / \mathrm{ml})$ immediately after infection. Three and $24 \mathrm{~h}$ after infection intracellular bacteria were enumerated. (A,B) RML LVS is more resistant to IFN- $\gamma$ mediated killing compared to all other strains tested. Deletion of the feoB gene rendered RML LVS more sensitive to IFN- $\gamma$ mediated killing and complementation with homologous feoB, but not ATCC feoB partially restored resistance to IFN- $\gamma$. (C,D) Deletion of the bacterioferritin (bfr) also increased RML LVS sensitivity to IFN- $\gamma$ mediated killing. Error bars represent SD. Significance was tested via two-way ANOVA followed by Tukey's multiple comparison of means. Significance was set at $p<$ 0.05. *, significantly greater than all other samples. ${ }^{* \star}$, significantly greater than RML $\Delta f e o B$. ${ }^{\star \star \star}$, significantly less than RML LVS. Data is representative of three experiments of similar design.

\section{DISCUSSION}

Francisella tularensis is a highly pathogenic bacterium for which no effective licensed vaccine exists. Due to concerns that the extreme pathogenicity of the organism might lead to its intentional release there is increased interest in developing an effective vaccine for F. tularensis infections. A complication with the LVS is that the genetic mutations that attenuated LVS are undefined, leaving open the possibility that the strain could revert to full virulence. While LVS is unlikely to be reapproved for human vaccination, it is used extensively as a standard by which other candidate Francisella vaccines can be measured in animal models, as well as a tool to discover correlates of immunity to $F$. tularensis. Indeed, in a previous report it was found that the RML LVS biovar stimulated protective immunity in a mouse model of vaccine against virulent $F$. tularensis Schu S4 by inducing higher numbers of effector $\mathrm{T}$ cells (Griffin et al., 2015). Building on this observation, we have sought to identify the genetic differences between biovars of LVS and explore how they may affect the outcomes of virulence and immunity in mouse models of infection. In this report, we have demonstrated that there is a significant difference between the
RML LVS and the Iowa LVS or ATCC LVS biovars in ferrous iron homeostasis.

Iron is one of the most abundant metals on the planet but is usually found in the environment in an insoluble oxidized state, which has required bacteria to develop mechanisms to scavenge iron from their local environment (Crichton and Pierre, 2001). The need for bacterial pathogens to acquire iron within a host environment presents additional challenges to the microorganism as they must also contend with host responses, which include mounting an immune response and sequestering iron, both of which can severely limit the ability of the pathogen to grow within the host (Cassat and Skaar, 2013). As expected, F. tularensis requires iron for productive infection of host cells, as mutants in ferrous and ferric iron uptake systems in $F$. tularensis LVS and F. tularensis Schu S4 were incapable of intracellular growth in vitro and were attenuated for virulence in mice (Perez and Ramakrishnan, 2014; Perez et al., 2016). Other work has shown that F. tularensis significantly increases expression of host iron-related genes, including transferrin receptor, presumably to increase the labile iron pools in the cytosol of infected host cells (Pan et al., 2010). Although F. tularensis requires iron for growth in a host, it appears to be 


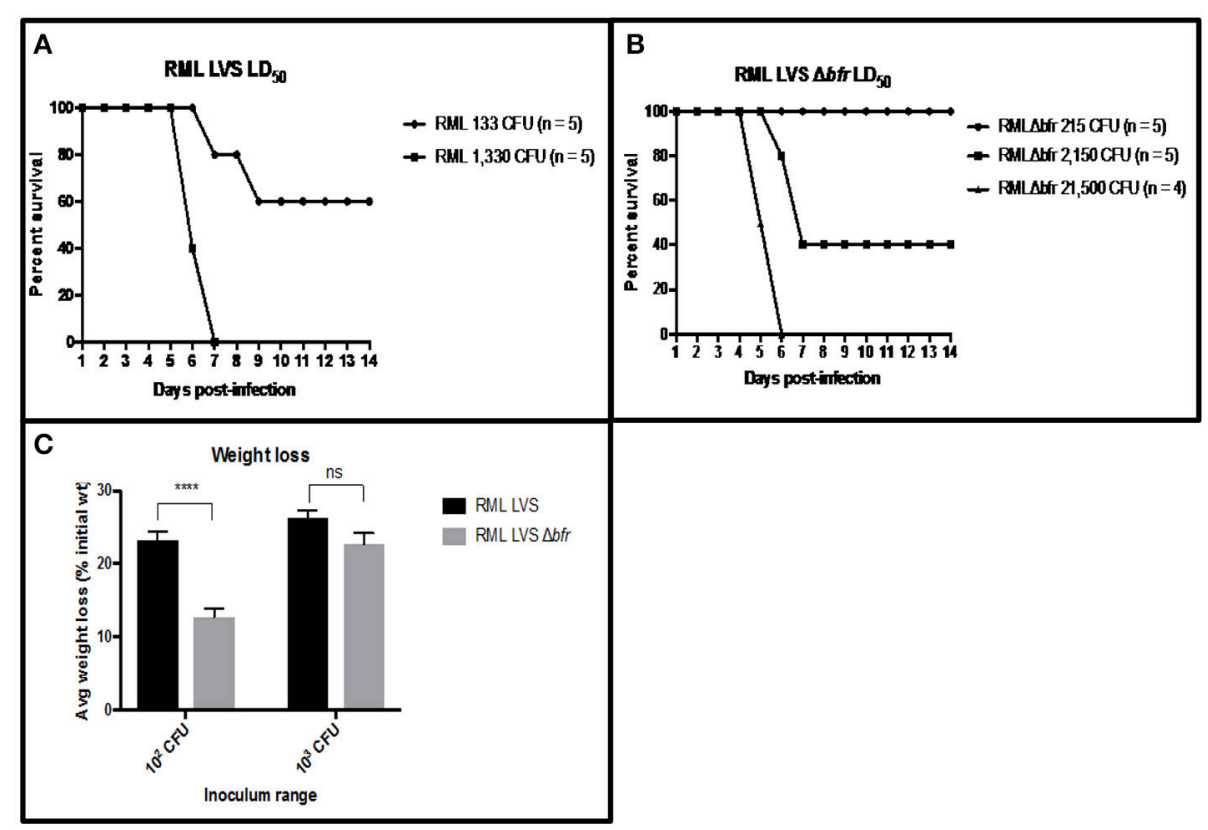

FIGURE 6 | Bacterioferritin is required for optimal RML LVS virulence and. (A) Groups of mice $(n=5)$ were intranasally infected with $25 \mu$ linocula containing 133 or 1,330 CFU of RML LVS in PBS. Mice were weighed daily and humanely sacrificed after weight loss $\geq 25 \%$ of their initial weight. $L D_{50}$ values were calculated via the method of Reed and Muench (1938). (B) Groups of mice $(n=5)$ were intranasally infected with 25 ul inocular containing 215, 2,150 or 21,500 CFU of RML LVS $\Delta b$ rf in PBS. Mice were weighed daily and humanely sacrificed after weight loss $\geq 25 \%$ of their initial weight. $L_{50}$ values were calculated via the method of Reed and Muench (1938). (C) The average weight loss of mice infected with $10^{2}$ or $10^{3}$ of the RML LVS strain or $10^{2}$ or $10^{3}$ of the RML LVS $\Delta b f r$ is represented by the bars in the graph. The significance of the weight loss of the mice infected with the RML LVS strain was compared to the weight loss of the mice infected with the RML LVS $\Delta$ bfr strain Mice infected with RML LVS were closer to or at clinical endpoint at all doses, while mice infected with RML LVS $\Delta$ bfr lost significantly less weight at the lowest infectious dose. Significance was set at $p<0.05$. ${ }^{\star \star \star \star}$ Indicates a significant difference between the two samples bracketed. Significance was tested via log-rank sum test.

\section{Challenge with 25 CFU F. tularensis Schu S4}

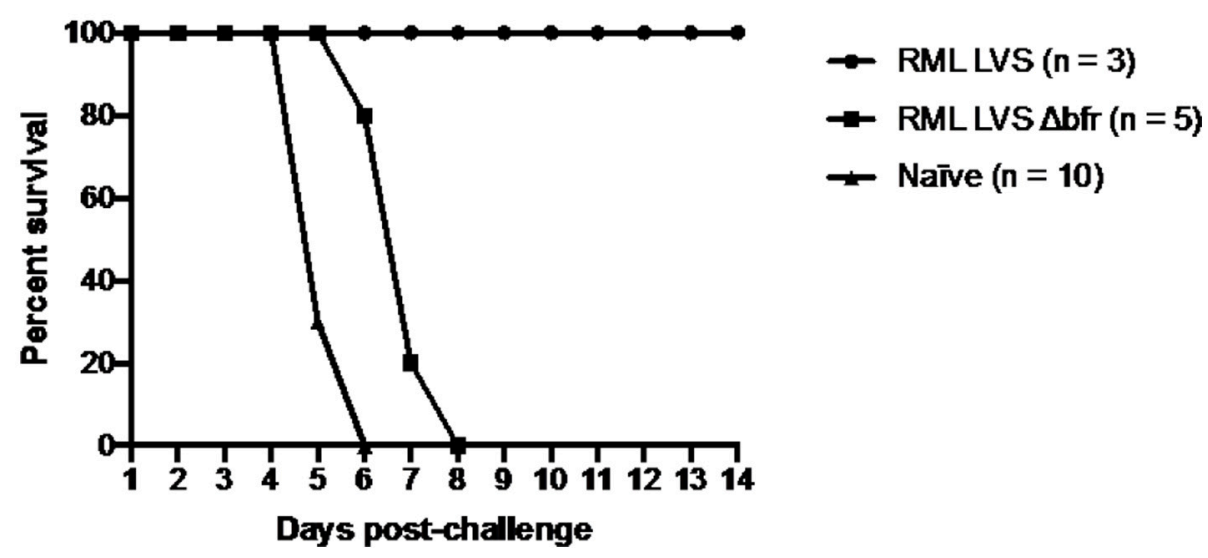

FIGURE 7 | RML requires bacterioferritin to elicit protection against an intranasal challenge of 25 CFU F. tularensis Schu S4. Mice were infected with either 121 CFU of RML LVS or $215 \mathrm{CFU}$ of the bacterioferritin mutant and allowed to recover for fix weeks before challenge. The challenge inoculum was suspended in $25 \mu$ l sterile PBS and administered intranasally. Health was monitored for the duration of infection and mice were humanely sacrificed if $\geq 25 \%$ of initial weight was lost. Data shown is from one replicate. Significance was tested via log-rank sum test.

unusual among bacterial pathogens in that its virulence may depend, in part, on maintaining optimally low levels of iron within its cytoplasm, as the highly virulent subsp. tularensis strains had approximately 4 - to 5 -fold less intracellular iron than the moderately virulent subsp. holarctica strains (Lindgren et al., 2011). 
Consistent with this model of Francisella virulence, we have shown that the more virulent RML LVS biovar encodes a nonsynonymous mutation in the $f e o B$ gene that confers phenotypes consistent with lower ferrous iron levels. The strain grows similarly to the Iowa and ATCC LVS biovars in standard propagation media, yet is restricted for growth when the iron concentrations are significantly reduced. This indicates that when extracellular iron levels become limiting, the RML LVS may not be as proficient at scavenging iron (via the $f e o B$ system) as Iowa LVS and ATCC LVS. Numerous labs have reported that expression of the $f_{s} l$ iron uptake operon genes are tightly regulated (repressed in high iron, induced in low iron) by iron

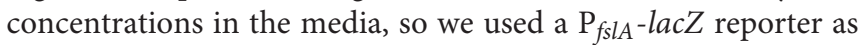
an indirect measure of relative ferrous iron levels between the various LVS biovars (Deng et al., 2006; Ramakrishnan et al., 2008; Lindgren et al., 2009). As with growth phenotypes, there were no detectable differences in $\mathrm{P}_{f_{s l A}}$-lac $Z$ expression in growth media with normal iron concentrations; however, the RML LVS had increased reporter activity (consistent with lower intracellular iron levels) relative to the Iowa LVS and ATCC LVS after growth in iron limiting media. We probed $f e o B$ function of the various LVS strains further by performing functional complementation experiments. Using a $E$. coli $\Delta f e o B$ mutant with a Fur-repressed fhuF-lacZ reporter as the complementation strain, we observed that the RML LVS $f e o B$ allele did not mediate repression of the fhuF-lacZ reporter (low intracellular iron), while both the Iowa LVS and ATCC LVS alleles did (high intracellular iron concentrations). Collectively, these experimental results provide compelling genetic evidence that the RML LVS likely has lower basal levels of intracellular ferrous iron than the Iowa LVS and ATCC LVS biovars. To our knowledge this is the first direct genetic evidence that explains differences in iron content between F. tularensis strains, though it is likely unique to LVS biovars. A significant contribution of this work is that it helps to provide a rationale for why virulent $F$. tularensis strains may carefully control their levels of intracellular iron as it appears that they balance the requirement of iron for growth against the effects of the Fenton reaction, which may induce oxidant damage to the bacteria that could expose the organisms to the host immune response (Binesse et al., 2015).

The low ferrous iron phenotypes exhibited by subsp. tularensis have been reported to correlate with resistance to the lethal effects of $\mathrm{H}_{2} \mathrm{O}_{2}$, so we sought to characterize the relative sensitivity of each LVS biovar in vitro. Indeed, the RML LVS exhibited nearly ten-fold more resistance to hydrogen peroxide than the Iowa LVS, which encodes a functional FeoB, and 100-fold or more resistance than the ATCC LVS. The latter strain is significantly more sensitive to $\mathrm{H}_{2} \mathrm{O}_{2}$ as it is competent for $\mathrm{Fe}^{2+}$ uptake through its functional $\mathrm{FeoB}$, and because it encodes a Dyp peroxidase lacking 31 amino acids near the C-terminal portion of the protein. Mutation of the $d y p$ gene has been shown previously to increase $\mathrm{H}_{2} \mathrm{O}_{2}$ sensitivity (Binesse et al., 2015), and a clean deletion of $d y p$ in the Iowa LVS recapitulates this phenotype (unpublished data). We found that the RML LVS became significantly more sensitive to $\mathrm{H}_{2} \mathrm{O}_{2}$ by introducing a functional $f e o B$ allele under constitutive expression on a plasmid; the $\mathrm{H}_{2} \mathrm{O}_{2}$ sensitivity was significantly lower when the nonfunctional RML allele was introduced into the strain. We further demonstrated that the increased $\mathrm{H}_{2} \mathrm{O}_{2}$ lethality associated with overexpression of a functional $f e o B$ was not unique to the RML LVS genetic background, as an Iowa LVS $\triangle f e o B$ mutant complemented with the mutant allele was more resistant to $\mathrm{H}_{2} \mathrm{O}_{2}$ than that complemented with the functional allele. These experiments are significant, as the strains only differed by a single nucleotide in $f e o B$, yet exhibited nearly ten-fold differences in their sensitivities to $\mathrm{H}_{2} \mathrm{O}_{2}$, irrespective of genetic background. In sum, these experiments strongly suggest that RML LVS is better at avoiding $\mathrm{Fe}^{2+}$-associated toxicity in the context of the host environment virtue due to its lower functioning FeoB system.

In addition to characterizing the $f e o B$ D471Y mutation in RML LVS, we also characterized a mutant lacking the putative iron storage protein bacterioferritin. Bacterioferritin is known to oxidize ferrous iron and store the ferric oxide mineral in the hollow cavity formed by a 24-mer sphere in other organisms (Honarmand Ebrahimi et al., 2015). We reasoned that a $F$. tularensis RML LVS mutant lacking bacterioferritin may be unable to sequester free $\mathrm{Fe}^{2+}$ as effectively as the wild type strain, and that this would be reflected in iron-related gene expression. The $\mathrm{P}_{f_{s l A}}$-lac $Z$ reporter indeed showed a modest decrease in expression in the $\Delta b f r$ mutant, indicating that the strain may have more $\mathrm{Fe}^{2+}$ available to interact with Fur and repress expression of the reporter. This result is consistent with the hypothesis that $F$. tularensis LVS uses bacterioferritin to alter the pools of intracellular $\mathrm{Fe}^{2+}$ levels by sequestration. The $\mathrm{P}_{f s l A}$-lac $Z$ reporter expression decreased by only $\sim 20 \%$ suggesting that the bacteria may utilize other iron storage mechanisms as well. As the iron-regulated reporter indicated that the $\Delta b f r$ mutant may have increased levels of intracellular ferrous iron, we hypothesized that the strain would be more sensitive to $\mathrm{H}_{2} \mathrm{O}_{2}$. We found that the $\Delta b f r$ mutant had approximately $\sim 90 \%$ reduction in viability relative to the parental strain, indicating that bacterioferritin has a role in resistance to oxidative stress in F. tularensis LVS.

As the plasmid-based complementation strategy for the Iowa LVS $\Delta f e o B$ was unsuitable for in vivo studies, we utilized the RML LVS $\Delta b f r$ mutant as an alternative for proof of principle, given

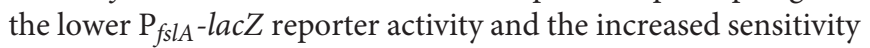
to $\mathrm{H}_{2} \mathrm{O}_{2}$ of this mutant. Intranasal infections of mice confirmed that bacterioferritin was required for full virulence of the strain, with the $\mathrm{LD}_{50}$ of the mutant estimated to be $\sim 1,500 \mathrm{CFU}$, nearly an eight-fold increase relative to the parental strain $(<200$ CFU). Additionally, the mice exhibited differences in the severity of disease, as mice infected with 133 CFU of RML LVS lost considerable weight during the infection, approximately twentythree percent on average. In contrast, the mice infected with 215 CFU of the $\Delta b f r$ mutant lost significantly less body weight during infection $(\sim 12 \%)$, even though the dose was almost two times higher. Only at a ten-fold higher dose of the $\Delta b f r$ mutant did the mice lose similar amounts of weight as those infected with the lowest dose of the parent strain.

As the RML LVS has been shown to be more effective at stimulating a protective response to challenge with $F$. tularensis Schu $\mathrm{S} 4$ than the more $\mathrm{H}_{2} \mathrm{O}_{2}$ sensitive biovar of LVS (ATCC), we hypothesized that loss of $\mathrm{H}_{2} \mathrm{O}_{2}$ resistance would render it a poor vaccine strain. Consistent with our hypothesis, the RML 
LVS $\Delta b f r$ did not stimulate robust protection in mice infected with this strain, as all succumbed from Schu S4 infection by day eight. These data suggest that $\mathrm{H}_{2} \mathrm{O}_{2}$ resistance may contribute to the efficacy of the RML LVS as a vaccine against tularemia in mice; however, it is possible that other interpretations of the data are valid. For example, the bacterioferritin protein is known to stimulate antibody production and $\mathrm{T}$ cell proliferation in LVS immunized mice, however it isn't clear that antibody responses are effective at controlling infection with virulent $F$. tularensis Schu S4 (Bakshi et al., 2008). In in vitro studies, F. tularensis Schu S4 is able to bind the active form of the host protease plasmin, which can cleave Francisella specific antibodies to avoid the ensuing bactericidal and inflammatory consequences of phagocytosis via antibody mediated opsonization (Crane et al., 2009). Furthermore, introduction of monoclonal antibodies against Francisella bacterioferritin into mice did not provide protection against lethal LVS challenge, even though other antibodies can be highly effective at protecting against this strain (Cole et al., 2009; Savitt et al., 2009). Taken together, these observations suggest that the lack of a bacterioferritin-specific antibody response is not likely to explain the poor efficacy of the RML LVS $\Delta b f r$ at protecting against Schu S4.

This work is significant for several reasons. The observation that the RML LVS is better able to induce host immunity than other LVS strains and has phenotypes consistent with relatively lower levels of free ferrous iron indicates that there is an important inverse correlation between the two. This finding is of particular importance for research groups using F. tularensis LVS as a model organism for studying tularemia. Care should be taken as lab workers plate LVS cultures during routine work to not overpassage LVS and/or inadvertently select for isolates that carry mutations that lead to higher acquisition of iron. This may have the unintended consequence of working with LVS isolates that have reduced immunogenicity and/or virulence. In addition, our findings highlight further the virulence strategy of F. tularensis which appears to be optimizing its ability to grow while avoiding, as much as possible, its visibility to the host's immune surveillance systems. Presumably, this contributes significantly to the extreme virulence of this bacterial pathogen. Finally, we believe that this

\section{REFERENCES}

Andrews, S. C., Robinson, A. K., and Rodriguez-Quinones, F. (2003). Bacterial iron homeostasis. FEMS Microbiol. Rev. 27, 215-237. doi: 10.1016/S0168-6445(03)00055-X

Aranda, J., Cortés, M. P., Garrido, M. E., Fittipaldi, N., Llagostera, M., Gottschalk, M., et al. (2009). Contribution of the FeoB transporter to Streptococcus suis virulence. Int. Microbiol. 12, 137-143. doi: 10.2436/20.1501.01.91

Ashkenazy, H., Erez, E., Martz, E., Pupko, T., and Ben-Tal, N. (2010). ConSurf 2010: calculating evolutionary conservation in sequence and structure of proteins and nucleic acids. Nucleic Acids Res. 38, W529-W533. doi: 10.1093/nar/gkq399

Bakshi, C. S., Malik, M., Mahawar, M., Kirimanjeswara, G. S., Hazlett, K. R., Palmer, L. E., et al. (2008). An improved vaccine for prevention of respiratory tularemia caused by Francisella tularensis SchuS4 strain. Vaccine 26, 5276-5288. doi: 10.1016/j.vaccine.2008.07.051

Bakshi, C. S., Malik, M., Regan, K., Melendez, J. A., Metzger, D. W., Pavlov, V. M., et al. (2006). Superoxide dismutase B gene (sodB)-deficient mutants work provides new information that should help to guide efforts to design a rational vaccine for tularemia.

\section{ETHICS STATEMENT}

All experiments using recombinant DNA techniques or reagents with F. tularensis LVS were approved by the Institutional Biosafety Committee. All animals were handled in strict accordance with good animal practice as defined by the relevant national and/or local animal welfare bodies, and all animal work was approved by the University of Iowa Animal Care and Use committee (ACURF \#1305086). Guidelines provided by the NIH were followed in all experimentation. The University of Iowa is PHS assured.

\section{AUTHOR CONTRIBUTIONS}

JF designed and performed a majority of experiments and was responsible for writing the manuscript. DC and TW designed and performed experiments in which LVS strains were used to infect macrophages and stimulate cytokine production. They also participated in editing the manuscript. CM performed bioinformatics analysis of the genomic sequences of strains and was responsible for editing the manuscript. $\mathrm{CB}$ oversaw experiments in her lab, she is responsible for the first observation of virulence differences in LVS strains and she edited and helped to write the manuscript. BJ oversaw research work performed by JF in his lab, is responsible for the final state of the manuscript and is the corresponding author.

\section{FUNDING}

This work was supported by the Intramural Research Program of the National Institutes of Health, National Institute of Allergy and Infectious Diseases (the lab of $\mathrm{CB}$ and the lab of $\mathrm{CM}$ ). Financial support for this work was provided to the lab BJ (NIH grants 2PO1 AI044642 and 2U54 AI057160). JF was supported by the Predoctoral Training Program in Genetics 5 T32 GM008629. of Francisella tularensis demonstrate hypersensitivity to oxidative stress and attenuated virulence. J. Bacteriol. 188, 6443-6448. doi: 10.1128/JB.00266-06

Binesse, J., Lindgren, H., Lindgren, L., Conlan, W., and Sjostedt, A. (2015). Roles of reactive oxygen species-degrading enzymes of Francisella tularensis SCHU S4. Infect. Immun. 83, 2255-2263. doi: 10.1128/IAI.02488-14

Bou-Abdallah, F., Lewin, A. C., Le Brun, N. E., Moore, G. R., and Chasteen, N. D. (2002). Iron detoxification properties of Escherichia coli bacterioferritin. Attenuation of oxyradical chemistry. J. Biol. Chem. 277, 37064-37069. doi: 10.1074/jbc.M205712200

Buchan, B. W., McCaffrey, R. L., Lindemann, S. R., Allen, L. A., and Jones, B. D. (2009). Identification of migR, a regulatory element of the Francisella tularensis live vaccine strain iglABCD virulence operon required for normal replication and trafficking in macrophages. Infect. Immun. 77, 2517-2529. doi: 10.1128/IAI.00229-09

Buchan, B. W., McLendon, M. K., and Jones, B. D. (2008). Identification of differentially regulated francisella tularensis genes by use of a newly developed Tn5-based transposon delivery system. Appl. Environ. Microbiol. 74, 2637-2645. doi: 10.1128/AEM.02882-07 
Cartron, M. L., Maddocks, S., Gillingham, P., Craven, C. J., and Andrews, S. C. (2006). Feo-transport of ferrous iron into bacteria. Biometals 19, 143-157. doi: 10.1007/s10534-006-0003-2

Cassat, J. E., and Skaar, E. P. (2013). Iron in infection and immunity. Cell Host Microbe 13, 509-519. doi: 10.1016/j.chom.2013.04.010

Cole, L. E., Yang, Y., Elkins, K. L., Fernandez, E. T., Qureshi, N., Shlomchik, M. J., et al. (2009). Antigen-specific B-1a antibodies induced by Francisella tularensis LPS provide long-term protection against $F$. tularensis LVS challenge. Proc. Natl. Acad. Sci. U.S.A. 106, 4343-4348. doi: 10.1073/pnas.0813411106

Crane, D. D., Bauler, T. J., Wehrly, T. D., and Bosio, C. M. (2014). Mitochondrial ROS potentiates indirect activation of the AIM2 inflammasome. Front. Microbiol. 5:438. doi: 10.3389/fmicb.2014.00438

Crane, D. D., Warner, S. L., and Bosio, C. M. (2009). A novel role for plasminmediated degradation of opsonizing antibody in the evasion of host immunity by virulent, but not attenuated, Francisella tularensis. J. Immunol. 183, 4593-4600. doi: 10.4049/jimmunol.0901655

Crichton, R. R., and Pierre, J. L. (2001). Old iron, young copper: from Mars to Venus. Biometals 14, 99-112. doi: 10.1023/A:1016710810701

Deng, K., Blick, R. J., Liu, W., and Hansen, E. J. (2006). Identification of Francisella tularensis genes affected by iron limitation. Infect. Immun. 74, 4224-4236. doi: 10.1128/IAI.01975-05

Edwards, J. A., Rockx-Brouwer, D., Nair, V., and Celli, J. (2010). Restricted cytosolic growth of Francisella tularensis subsp. tularensis by IFN-gamma activation of macrophages. Microbiology 156, 327-339. doi: $10.1099 /$ mic. $0.031716-0$

Eigelsbach, H. T., and Downs, C. M. (1961). Prophylactic effectiveness of live and killed tularemia vaccines. I. Production of vaccine and evaluation in the white mouse and guinea pig. J. Immunol. 87, 415-425.

Griffin, A. J., Crane, D. D., Wehrly, T. D., and Bosio, C. M. (2015). Successful protection against tularemia in C57BL/6 mice is correlated with expansion of Francisella tularensis-specific effector T cells. Clin. Vaccine Immunol. 22, 119-128. doi: 10.1128/CVI.00648-14

Hantke, K. (2003). Is the bacterial ferrous iron transporter FeoB a living fossil? Trends Microbiol. 11, 192-195. doi: 10.1016/S0966-842X(03)00100-8

Honarmand Ebrahimi, K., Hagedoorn, P. L., and Hagen, W. R. (2015). Unity in the biochemistry of the iron-storage proteins ferritin and bacterioferritin. Chem. Rev. 115, 295-326. doi: 10.1021/cr5004908

Imlay, J. A. (2013). The molecular mechanisms and physiological consequences of oxidative stress: lessons from a model bacterium. Nat. Rev. Microbiol 11, 443-454. doi: 10.1038/nrmicro3032

Kammler, M., Schon, C., and Hantke, K. (1993). Characterization of the ferrous iron uptake system of Escherichia coli. J. Bacteriol. 175, 6212-6219. doi: 10.1128/jb.175.19.6212-6219.1993

Kim, H., Lee, H., and Shin, D. (2012). The FeoA protein is necessary for the FeoB transporter to import ferrous iron. Biochem. Biophys. Res. Commun. 423, 733-738. doi: 10.1016/j.bbrc.2012.06.027

Landau, M., Mayrose, I., Rosenberg, Y., Glaser, F., Martz, E., Pupko, T., et al. (2005). ConSurf 2005: the projection of evolutionary conservation scores of residues on protein structures. Nucleic Acids Res. 33, W299-W302. doi: $10.1093 /$ nar/gki370

Langmead, B., and Salzberg, S. L. (2012). Fast gapped-read alignment with Bowtie 2. Nat. Methods 9, 357-359. doi: 10.1038/nmeth.1923

Lau, C. K., Ishida, H., Liu, Z., and Vogel, H. J. (2013). Solution structure of Escherichia coli FeoA and its potential role in bacterial ferrous iron transport. J. Bacteriol. 195, 46-55. doi: 10.1128/JB.01121-12

Lindemann, S. R., Peng, K., Long, M. E., Hunt, J. R., Apicella, M. A., Monack, D. M., et al. (2011). Francisella tularensis Schu S4 O-antigen and capsule biosynthesis gene mutants induce early cell death in human macrophages. Infect. Immun. 79, 581-594. doi: 10.1128/IAI.00863-10

Lindgren, H., Honn, M., Golovlev, I., Kadzhaev, K., Conlan, W., and Sjöstedt, A. (2009). The 58-kilodalton major virulence factor of Francisella tularensis is required for efficient utilization of iron. Infect. Immun. 77, 4429-4436. doi: 10.1128/IAI.00702-09

Lindgren, H., Honn, M., Salomonsson, E., Kuoppa, K., Forsberg, Å., and Sjöstedt, A. (2011). Iron content differs between Francisella tularensis subspecies tularensis and subspecies holarctica strains and correlates to their susceptibility to $\mathrm{H}_{2} \mathrm{O}_{2}$-induced killing. Infect. Immun. 79, 1218-1224. doi: 10.1128/IAI.01116-10
Lindgren, H., Lindgren, L., Golovliov, I., and Sjostedt, A. (2015). Mechanisms of heme utilization by Francisella tularensis. PLoS ONE 10:e0119143. doi: 10.1371/journal.pone.0119143

Lindgren, H., Shen, H., Zingmark, C., Golovliov, I., Conlan, W., and Sjöstedt, A. (2007). Resistance of Francisella tularensis strains against reactive nitrogen and oxygen species with special reference to the role of KatG. Infect. Immun. 75, 1303-1309. doi: 10.1128/IAI.01717-06

Llewellyn, A. C., Jones, C. L., Napier, B. A., Bina, J. E., and Weiss, D. S. (2011). Macrophage replication screen identifies a novel Francisella hydroperoxide resistance protein involved in virulence. PLOS ONE 6:e24201. doi: 10.1371/journal.pone.0024201

Ma, Z., Banik, S., Rane, H., Mora, V. T., Rabadi, S. M., Doyle, C. R., et al. (2014). EmrA1 membrane fusion protein of Francisella tularensis LVS is required for resistance to oxidative stress, intramacrophage survival and virulence in mice. Mol. Microbiol. 91, 976-995. doi: 10.1111/mmi.12509

Marlovits, T. C., Haase, W., Herrmann, C., Aller, S. G., and Unger, V. M. (2002). The membrane protein FeoB contains an intramolecular $\mathrm{G}$ protein essential for Fe(II) uptake in bacteria. Proc. Natl. Acad. Sci. U.S.A. 99, 16243-16248. doi: $10.1073 /$ pnas. 242338299

McCaffrey, R. L., Schwartz, J. T., Lindemann, S. R., Moreland, J. G., Buchan, B. W., Jones, B. D., et al. (2010). Multiple mechanisms of NADPH oxidase inhibition by type A and type B Francisella tularensis. J. Leukoc. Biol. 88, 791-805. doi: 10.1189/jlb.1209811

McKenna, A., Hanna, M., Banks, E., Sivachenko, A., Cibulskis, K., Kernytsky, A., et al. (2010). The genome analysis toolkit: a MapReduce framework for analyzing next-generation DNA sequencing data. Genome Res. 20, 1297-1303. doi: $10.1101 /$ gr. 107524.110

Melillo, A. A., Bakshi, C. S., and Melendez, J. A. (2010). Francisella tularensis antioxidants harness reactive oxygen species to restrict macrophage signaling and cytokine production. J. Biol. Chem. 285, 27553-27560. doi: 10.1074/jbc.M110.144394

Melillo, A. A., Mahawar, M., Sellati, T. J., Malik, M., Metzger, D. W., Melendez, J. A., et al. (2009). Identification of Francisella tularensis live vaccine strain CuZn superoxide dismutase as critical for resistance to extracellularly generated reactive oxygen species. J. Bacteriol. 191, 6447-6456. doi: 10.1128/JB.00534-09

Nagy, T. A., Moreland, S. M., and Detweiler, C. S. (2014). Salmonella acquires ferrous iron from haemophagocytic macrophages. Mol. Microbiol. 93, 1314-1326. doi: 10.1111/mmi.12739

Naikare, H., Palyada, K., Panciera, R., Marlow, D., and Stintzi, A. (2006). Major role for FeoB in Campylobacter jejuni ferrous iron acquisition, gut colonization, and intracellular survival. Infect. Immun. 74, 5433-5444. doi: 10.1128/IAI.00052-06

Pan, X., Tamilselvam, B., Hansen, E. J., and Daefler, S. (2010). Modulation of iron homeostasis in macrophages by bacterial intracellular pathogens. BMC Microbiol. 10:64. doi: 10.1186/1471-2180-10-64

Parrow, N. L., Fleming, R. E., and Minnick, M. F. (2013). Sequestration and scavenging of iron in infection. Infect. Immun. 81, 3503-3514. doi: 10.1128/IAI.00602-13

Perez, N., Johnson, R., Sen, B., and Ramakrishnan, G. (2016). Two parallel pathways for ferric and ferrous iron acquisition support growth and virulence of the intracellular pathogen Francisella tularensis Schu S4. Microbiologyopen 5, 453-468. doi: 10.1002/mbo3.342

Perez, N. M., and Ramakrishnan, G. (2014). The reduced genome of the Francisella tularensis live vaccine strain (LVS) encodes two iron acquisition systems essential for optimal growth and virulence. PLOS ONE 9:e93558. doi: 10.1371/journal.pone.0093558

Rabadi, S. M., Sanchez, B. C., Varanat, M., Ma, Z., Catlett, S. V., Melendez, J. A., et al. (2015). Antioxidant defenses of Francisella tularensis modulate macrophage function and production of proinflammatory cytokines. J. Biol. Chem. 291, 5009-5021. doi: 10.1074/jbc.M115.681478

Ramakrishnan, G., Meeker, A., and Dragulev, B. (2008). fslE is necessary for siderophore-mediated iron acquisition in Francisella tularensis Schu S4. J. Bacteriol. 190, 5353-5361. doi: 10.1128/JB.00181-08

Ramakrishnan, G., and Sen, B. (2014). The FupA/B protein uniquely facilitates transport of ferrous iron and siderophore-associated ferric iron across the outer membrane of Francisella tularensis live vaccine strain. Microbiology 160, 446-457. doi: 10.1099/mic.0.072835-0

Ramond, E., Gesbert, G., Rigard, M., Dairou, J., Dupuis, M., Dubail, I., et al. (2014). Glutamate utilization couples oxidative stress defense and the tricarboxylic 
acid cycle in Francisella phagosomal escape. PLoS Pathog. 10:e1003893. doi: 10.1371/journal.ppat.1003893

Reed, L. J., and Muench, H. (1938). A simple method of estimating fifty percent endpoints. Am. J. Hygiene 27, 493-497.

Robey, M., and Cianciotto, N. P. (2002). Legionella pneumophila feoAB promotes ferrous iron uptake and intracellular infection. Infect. Immun. 70, 5659-5669. doi: 10.1128/IAI.70.10.5659-5669.2002

Rohmer, L., Fong, C., Abmayr, S., Wasnick, M., Larson Freeman, T. J., Radey, M., et al. (2007). Comparison of Francisella tularensis genomes reveals evolutionary events associated with the emergence of human pathogenic strains. Genome Biol. 8:R102. doi: 10.1186/gb-2007-8-6-r102

Rohmer, L., Brittnacher, M., Svensson, K., Buckley, D., Haugen, E., Zhou, Y., et al. (2006). Potential source of Francisella tularensis live vaccine strain attenuation determined by genome comparison. Infect. Immun. 74, 6895-6906. doi: 10.1128/IAI.01006-06

Salomonsson, E., Kuoppa, K., Forslund, A. L., Zingmark, C., Golovliov, I., Sjöstedt, A., et al. (2009). Reintroduction of two deleted virulence loci restores full virulence to the live vaccine strain of Francisella tularensis. Infect. Immun. 77, 3424-3431. doi: 10.1128/IAI.00196-09

Savitt, A. G., Mena-Taboada, P., Monsalve, G., and Benach, J. L. (2009). Francisella tularensis infection-derived monoclonal antibodies provide detection, protection, and therapy. Clin. Vaccine Immunol. 16, 414-422. doi: 10.1128/CVI.00362-08

Schulert, G. S., McCaffrey, R. L., Buchan, B. W., Lindemann, S. R., Hollenback, C., Jones, B. D., et al. (2009). Francisella tularensis genes required for inhibition of the neutrophil respiratory burst and intramacrophage growth identified by random transposon mutagenesis of strain LVS. Infect. Immun. 77, 1324-1336. doi: 10.1128/IAI.01318-08

Shakerley, N. L., Chandrasekaran, A., Trebak, M., Miller, B. A., and Melendez, J. A. (2015). Francisella tularensis catalase restricts immune function by impairing TRPM2 channel activity. .J Biol. Chem. 291, 3871-3881. doi: 10.1074/jbc.M115.706879

Su, J., Yang, J., Zhao, D., Kawula, T. H., Banas, J. A., and Zhang, J. R. (2007). Genome-wide identification of Francisella tularensis virulence determinants. Infect. Immun. 75, 3089-3101. doi: 10.1128/IAI.01865-06

Su, Y. C., Chin, K. H., Hung, H. C., Shen, G. H., Wang, A. H., and Chou, S. H. (2010). Structure of Stenotrophomonas maltophilia FeoA complexed with zinc: a unique prokaryotic SH3-domain protein that possibly acts as a bacterial ferrous iron-transport activating factor. Acta Crystallogr. F Struct. Biol. Cryst. Commun. 66, 636-642. doi: 10.1107/S1744309110013941

Sullivan, J. T., Jeffery, E. F., Shannon, J. D., and Ramakrishnan, G. (2006). Characterization of the siderophore of Francisella tularensis and role of fslA in siderophore production. J. Bacteriol. 188, 3785-3795. doi: 10.1128/JB.00027-06

Thomas-Charles, C. A., Zheng, H. Palmer, L. E., Mena, P. Thanassi, D. G., and Furie M. B., et al. (2013). FeoB-mediated uptake of iron by Francisella tularensis. Infect. Immun. 81, 2828-2837. doi: 10.1128/IAI.00170-13

Troxell, B., and Hassan, H. M. (2013). Transcriptional regulation by Ferric Uptake Regulator (Fur) in pathogenic bacteria. Front. Cell. Infect. Microbiol. 3:59. doi: 10.3389/fcimb.2013.00059

Velayudhan, J., Hughes, N. J., McColm, A. A., Bagshaw, J., Clayton, C. L., Andrews, S. C., et al. (2000). Iron acquisition and virulence in Helicobacter pylori: a major role for FeoB, a high-affinity ferrous iron transporter. Mol. Microbiol. 37, 274-286. doi: 10.1046/j.1365-2958.2000.01987.x

Weaver, E. A., Wyckoff, E. E., Mey, A. R., Morrison, R., and Payne, S. M. (2013). $\mathrm{FeoA}$ and $\mathrm{FeoC}$ are essential components of the Vibrio cholerae ferrous iron uptake system, and FeoC interacts with FeoB. J. Bacteriol. 195, 4826-4835. doi: $10.1128 /$ JB.00738-13

Wehrly, T. D., Chong, A., Virtaneva, K., Sturdevant, D. E., Child, R., Edwards, J. A., et al. (2009). Intracellular biology and virulence determinants of Francisella tularensis revealed by transcriptional profiling inside macrophages. Cell. Microbiol. 11, 1128-1150. doi: 10.1111/j.1462-5822.2009.01316.x

Zubay, G., Morse, D. E., Schrenk, W. J., and Miller, J. H. (1972). Detection and isolation of the repressor protein for the tryptophan operon of Escherichia coli. Proc. Natl. Acad. Sci. U.S.A. 69, 1100-1103. doi: 10.1073/pnas.69.5.1100

Conflict of Interest Statement: The authors declare that the research was conducted in the absence of any commercial or financial relationships that could be construed as a potential conflict of interest.

Copyright (๑ 2018 Fletcher, Crane, Wehrly, Martens, Bosio and Jones. This is an open-access article distributed under the terms of the Creative Commons Attribution License (CC BY). The use, distribution or reproduction in other forums is permitted, provided the original author(s) and the copyright owner are credited and that the original publication in this journal is cited, in accordance with accepted academic practice. No use, distribution or reproduction is permitted which does not comply with these terms. 